Zhiwei Zhang*, Shujie Ma, Lei Nie and Guoxing Soon

\title{
A Quantitative Concordance Measure for Comparing and Combining Treatment Selection Markers
}

DOI 10.1515/ijb-2016-0064

\begin{abstract}
Motivated by an HIV example, we consider how to compare and combine treatment selection markers, which are essential to the notion of precision medicine. The current literature on precision medicine is focused on evaluating and optimizing treatment regimes, which can be obtained by dichotomizing treatment selection markers. In practice, treatment decisions are based not only on efficacy but also on safety, cost and individual preference, making it difficult to choose a single cutoff value for all patients in all settings. It is therefore desirable to have a statistical framework for comparing and combining treatment selection markers without dichotomization. We provide such a framework based on a quantitative concordance measure, which quantifies the extent to which higher marker values are predictive of larger treatment effects. For a given marker, the proposed concordance measure can be estimated from clinical trial data using a U-statistic, which can incorporate auxiliary covariate information through an augmentation term. For combining multiple markers, we propose to maximize the estimated concordance measure among a specified family of combination markers. A cross-validation procedure can be used to remove any re-substitution bias in assessing the quality of an optimized combination marker. The proposed methodology is applied to the HIV example and evaluated in simulation studies.
\end{abstract}

Keywords: cross-validation, personalized medicine, predictive biomarker, precision medicine, treatment effect heterogeneity, U-statistic

\section{Introduction}

It is well recognized that treatment effects can be heterogeneous; that is, the same treatment can have different effects on different patients. The increasing awareness of treatment effect heterogeneity has motivated the development of predictive biomarkers for treatment selection [1, 2]. When two or more markers are available for the same treatment selection problem, methods are needed for comparing different markers and for combining multiple markers into a single marker. Our interest in these problems is motivated by two randomized clinical trials known as ECHO [3] and THRIVE [4], which compared the antiretroviral drugs rilpivirine and efavirenz for treatment-naive adult patients infected with human immunodeficiency virus (HIV). Both drugs are non-nucleoside reverse transcriptase inhibitors to be used in combination with other antiretroviral agents. The ECHO and THRIVE trials differed mainly in the use of background nucleoside/nucleotide reverse transcriptase inhibitors (N[t]RTIs). Except for that difference, the two trials followed nearly identical designs, produced very similar results [5], and are therefore combined in our analysis. The combined trial data show that rilpivirine and efavirenz are of comparable efficacy with respect to virologic response (viral

*Corresponding author: Zhiwei Zhang, Department of Statistics, University of California, Riverside, CA, USA, E-mail: zhiwei.zhang@ucr.edu

Shujie Ma, Department of Statistics, University of California, Riverside, CA, USA

Lei Nie, Division of Biometrics V, Office of Biostatistics, Center for Drug Evaluation and Research, Food and Drug Administration, Silver Spring, MD, USA

Guoxing Soon, Division of Biometrics IV, Office of Biostatistics, Center for Drug Evaluation and Research, Food and Drug Administration, Silver Spring, MD, USA 

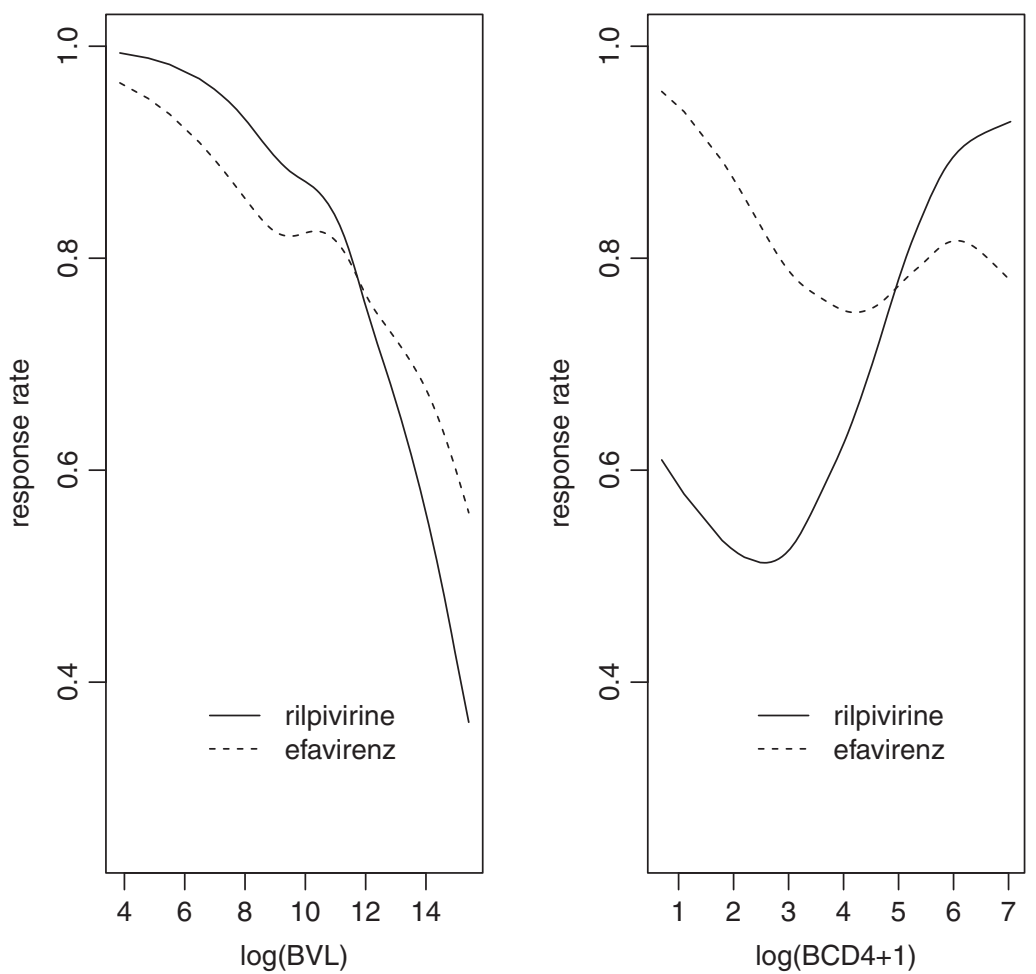

Figure 1: Nonparametric regression analysis of the combined HIV trial data: smoothed estimates of treatment-specific virologic response rates as functions of BVL (left) and BCD4 (right).

load $<50$ copies/ml at week 48 of treatment). Exploratory analyses suggest that the relative efficacy of rilpivirine and efavirenz may depend on baseline viral load (BVL) and baseline CD4 cell count (BCD4) [6, 7]. Figure 1 shows nonparametric estimates of treatment-specific virologic response rates as functions of marker value, separately for each marker. A logistic regression analysis with treatment and log-transformed marker values as covariates shows significant interactions between treatment and the two markers (likelihood ratio test $p=0.0018$ ). These analyses suggest that BVL and BCD4 may be useful for treatment selection, and in this article we consider how to compare the two markers and how to combine them into a single marker.

The problems we consider are related to but different from evaluating and optimizing treatment regimes. A continuous or ordinal marker for treatment selection can be dichotomized into a treatment regime, which has a direct impact on the target population. The selection impact curve of Song and Pepe [8] can be used to visualize the impact of the resulting regime as a function of the cutoff value. The existing literature on precision medicine includes a variety of methods for estimating or approximating optimal treatment regimes that maximize a summary measure of efficacy [e.g., 9-12]. In practice, treatment decisions are based not only on efficacy but also on safety, cost and individual preference, which makes it difficult to apply a single cutoff value to all patients in all settings [13]. In our application, for example, rilpivirine is thought to be safer than efavirenz, and different patients may assign different weights to efficacy, safety and cost. Without a strong consensus on how to choose a cutoff, it is desirable to have a statistical framework for comparing and combining treatment selection markers without dichotomization.

There is an interesting analogy here between treatment selection and diagnostic medicine. A continuous or ordinal diagnostic marker can, and eventually will, be dichotomized into a binary diagnostic test. The choice of cutoff value involves balancing the two types of misclassifications, whose relative importance may depend on individual preference and circumstance. Without assuming a specific cutoff value, a continuous or ordinal diagnostic marker can be evaluated using the receiver operating characteristic (ROC) curve [14, 15]. Two diagnostic markers on different scales can be compared with respect to the area under the ROC curve 
(AUC), and multiple diagnostic markers can be combined by maximizing the AUC for the combination marker [16-18].

In this article, we propose a quantitative concordance measure for comparing and combining multiple treatment selection markers. The proposed concordance measure quantifies the extent to which higher marker values are predictive of larger treatment effects, and is closely related to the area under the selection impact curve defined by Song and Pepe [8]. For a given treatment selection marker, the proposed concordance measure can be estimated from clinical trial data using a U-statistic, which can incorporate auxiliary covariate information through an augmentation term [e.g., 7, 19]. For combining multiple markers, we propose to maximize the estimated concordance measure among a specified family of combination markers. This is similar in spirit to the approach of Zhang et al. [11] for estimating optimal treatment regimes. When there are more than three markers to combine, it may be impractical to find the optimal combination through a grid search, but the objective function can be smoothed as in Ma and Huang [18] so that a gradient descent algorithm can be applied. In assessing the quality of the optimized combination marker, there can be a resubstitution bias if the same data are used to estimate and evaluate the optimal combination [1,20, 21], and we propose to remove the re-substitution bias using a cross-validation procedure.

\section{Methodology}

\subsection{A quantitative concordance measure}

Consider a clinical trial in which each patient receives a randomized treatment $T$ ( 1 experimental; -1 control). We assume for simplicity that $\mathrm{P}(T=1)=\mathrm{P}(T=-1)=1 / 2$, although the main ideas extend easily to different allocation probabilities. Let $Y^{(t)}$ denote the potential outcome that will realize if a patient receives treatment $t \in\{1,-1\}$ [22]. Assuming consistency or stable unit treatment value, the actual outcome is just $Y=Y^{(T)}$. Without loss of generality, we assume that higher values of $Y$ are desirable. Let $\boldsymbol{X}$ be a vector of baseline covariates including one or more markers that may be considered in choosing between the two treatments.

We start by considering how to assess the utility of one marker, say $V$, for treatment selection. To this end, it is important to consider the conditional effect of $t=1$ versus $t=-1$ given $V$, defined as

$$
\delta(V)=\mathrm{E}\left\{Y^{(1)}-Y^{(-1)} \mid V\right\} .
$$

For a useless marker, the conditional effect function is constant: $\delta(V) \equiv \mathrm{E}\left\{Y^{(1)}-Y^{(-1)}\right\}=: \Delta$. A useful marker should have a large amount of variation in the function $\delta$. We assume throughout that higher marker values are intended to predict larger treatment effects, which implies that only "positive" variation in $\delta$ is desirable. This motivates our definition of $y=E\left(G_{12}\right)$ with

$$
G_{12}=\operatorname{sgn}\left(V_{1}-V_{2}\right)\left\{\delta\left(V_{1}\right)-\delta\left(V_{2}\right)\right\},
$$

where $V_{1}$ and $V_{2}$ are the marker values of two independent patients, and sgn is the sign function: $\operatorname{sgn}(u)=1,0,-1$ according as $u>,=,<0$.

We call y a quantitative concordance measure because $G_{12}$ involves the actual difference $\delta\left(V_{1}\right)-\delta\left(V_{2}\right)$ and not just the sign of the difference. An analogous qualitative concordance measure might be defined as $\mathrm{y}^{*}=\mathrm{E}\left(G_{12}^{*}\right)$ with

$$
G_{12}^{*}=\operatorname{sgn}\left(V_{1}-V_{2}\right) \operatorname{sgn}\left\{\delta\left(V_{1}\right)-\delta\left(V_{2}\right)\right\}
$$

It is easy to see that $\gamma^{*}=1$ if $V$ is continuous and $\delta$ is strictly increasing. Among such markers, it is certainly desirable to identify those with large variation in $\delta$ while considering the distribution of $V$. That extra information is captured by the quantitative concordance measure $y$, which is therefore the focus of this article. 
To understand what $y$ means, suppose the two patients are to receive treatments 1 and -1 . If $V_{1}>V_{2}$, then

$$
\begin{aligned}
G_{12} & =\delta\left(V_{1}\right)-\delta\left(V_{2}\right) \\
& =\mathrm{E}\left\{Y_{1}^{(1)}-Y_{1}^{(-1)} \mid V_{1}\right\}-\mathrm{E}\left\{Y_{2}^{(1)}-Y_{2}^{(-1)} \mid V_{2}\right\} \\
& =\mathrm{E}\left\{Y_{1}^{(1)}+Y_{2}^{(-1)} \mid V_{1}, V_{2}\right\}-\mathrm{E}\left\{Y_{1}^{(-1)}+Y_{2}^{(1)} \mid V_{1}, V_{2}\right\},
\end{aligned}
$$

where the last step makes use of the independence of the two patients. If $V_{1}<V_{2}$, the same argument shows that

$$
G_{12}=\mathrm{E}\left\{Y_{1}^{(-1)}+Y_{2}^{(1)} \mid V_{1}, V_{2}\right\}-\mathrm{E}\left\{Y_{1}^{(1)}+Y_{2}^{(-1)} \mid V_{1}, V_{2}\right\}
$$

If $V_{1}=V_{2}$, then $G_{12}=0$. Thus, $G_{12}$ measures the impact of giving treatment 1 to the patient with a larger $V$-value (if there is one) versus the opposite treatment allocation, and y represents the population average impact of treatment allocation based on $V$ versus $-V$. A large value of $y$ is clearly desirable. Note also that $y$ is invariant under a strictly increasing transformation of the marker.

Further insights into y can be gained by considering the selection impact curve of Song and Pepe [8]. Assume for the moment that $V$ is continuously distributed on an interval. Without loss of generality, we further assume that $V$ is uniformly distributed on $(0,1)$. The selection impact curve is the function

$$
\theta(v)=v \mathrm{E}\left\{Y^{(-1)} \mid V<v\right\}+(1-v) \mathrm{E}\left\{Y^{(1)} \mid V>v\right\},
$$

which gives the mean outcome for a treatment regime that assigns treatment 1 to patients with $V>v$ and treatment -1 to everyone else. Without specifying a cutoff $v$, a natural summary of the selection impact curve is its integral over the unit interval, which is analogous to the AUC for an ROC curve. In Appendix A, we show that

$$
\mathrm{y}=4 \operatorname{cov}\{V, \delta(V)\}=4 \int_{0}^{1} \theta(v) \mathrm{d} v-2 \mathrm{E}\left\{Y^{(-1)}\right\}-2 \mathrm{E}\left\{Y^{(1)}\right\}
$$

Thus, for a given pair of treatments, $y$ is directly related to the area under the selection impact curve.

\subsection{Estimating $y$ for a given marker}

We now consider estimation of $y$ for a given marker using a random sample of $(\boldsymbol{X}, T, Y)$ (recall that $\boldsymbol{X}$ includes $V$ as a component), with individual subjects denoted by subscripts (e.g., $i=1, \ldots, n$ ). Estimation of $y$ is complicated by the fact that the conditional effect function $\delta$ is unknown. However, we note that

$$
\mathrm{E}(2 T Y \mid V)=2 \mathrm{P}(T=1 \mid V) \mathrm{E}\left\{Y^{(1)} \mid V\right\}-2 \mathrm{P}(T=-1 \mid V) \mathrm{E}\left\{Y^{(-1)} \mid V\right\}=\delta(V),
$$

which suggests that $\delta(V)$ may be estimated by $2 T Y$ [7, 19]. In fact, $\delta(V)$ could be estimated by $2 T(Y-A)$ for any $A=a(\boldsymbol{X})$, because $\mathrm{E}(T A \mid V)=\mathrm{E}\{\mathrm{E}(T A \mid \boldsymbol{X}) \mid V\}=0$ due to randomization. The inclusion of $A$ is motivated by a potential gain in efficiency, as will become clear later.

The foregoing discussion, together with the definition of $y$, suggests the following estimator:

$$
\widehat{\gamma}(a)=\frac{2}{n(n-1)} \sum_{i<j} \widehat{G}_{i j}(a) \text {, }
$$

where

$$
\widehat{G}_{i j}(a)=2 \operatorname{sgn}\left(V_{i}-V_{j}\right)\left\{T_{i}\left(Y_{i}-A_{i}\right)-T_{j}\left(Y_{j}-A_{j}\right)\right\} .
$$


Noting that $\widehat{y}(a)$ is a one-sample U-statistic of order 2, it follows from standard asymptotic theory [e.g., van der Vaart 23, Chapter 12] that $\sqrt{n}\{\widehat{y}(a)-\gamma\}$ converges to a normal distribution with mean zero and variance

$$
\sigma^{2}(a)=4 \operatorname{cov}\left\{\widehat{G}_{12}(a), \widehat{G}_{13}(a)\right\} .
$$

A consistent estimator of $\sigma^{2}(a)$ is given by

$$
\widehat{\sigma}^{2}(a)=\frac{8}{n(n-1)(n-2)} \sum_{j \neq i \neq k, j<k} \widehat{G}_{i j}(a) \widehat{G}_{i k}(a)-4 \widehat{\gamma}^{2}(a) .
$$

In Appendix B, we show that the asymptotic variance $\sigma^{2}(a)$ is minimized by taking $a(\boldsymbol{X})$ to be

$$
a_{\mathrm{opt}}(\boldsymbol{X})=\mathrm{E}(Y \mid \boldsymbol{X}) .
$$

To take advantage of this result, we can specify and estimate a working model $\mathrm{E}(Y \mid \boldsymbol{X})=m(\boldsymbol{X} ; \boldsymbol{\alpha})$, where $m$ is a known function and $\boldsymbol{\alpha}$ is an unknown finite-dimensional parameter. For example, $m(\boldsymbol{X} ; \boldsymbol{\alpha})$ may be specified as a generalized linear model, and $\boldsymbol{\alpha}$ may be estimated by (iteratively reweighted) least squares. Let $\widehat{\boldsymbol{\alpha}}$ denote the resulting estimate of $\boldsymbol{\alpha}$; then we can estimate $a_{\text {opt }}(\boldsymbol{X})$ by $\widehat{a}_{\text {opt }}(\boldsymbol{X})=m(\boldsymbol{X} ; \widehat{\boldsymbol{\alpha}})$. We show in Appendix B that, whether the model $m(\boldsymbol{X} ; \boldsymbol{\alpha})$ is correct or not, $\widehat{\gamma}\left(\widehat{a}_{\text {opt }}\right)$ is consistent for $\gamma$ and asymptotically normal, and its asymptotic variance is given by $\sigma^{2}\left(m\left(\cdot ; \boldsymbol{\alpha}_{\infty}\right)\right)$, where $\boldsymbol{\alpha}_{\infty}$ is the probability limit of $\widehat{\boldsymbol{\alpha}}$. If $m(\boldsymbol{X} ; \boldsymbol{\alpha})$ is correctly specified, then $\boldsymbol{\alpha}_{\infty}$ is the true value of $\boldsymbol{\alpha}$ and $\widehat{y}\left(\widehat{a}_{\text {opt }}\right)$ is asymptotically equivalent to $\widehat{y}\left(a_{\text {opt }}\right)$. Regardless of the (in)correctness of $m(\boldsymbol{X} ; \boldsymbol{\alpha})$, the asymptotic variance $\sigma^{2}\left(m\left(\cdot ; \boldsymbol{\alpha}_{\infty}\right)\right)$ is consistently estimated by $\widehat{\sigma}^{2}\left(\widehat{a}_{\text {opt }}\right)$.

\subsection{Comparing two markers}

We now consider the problem of comparing two markers, say $V_{b}$ and $V_{c}$, both of which are included in $\boldsymbol{X}$. Write $\gamma_{b}=\mathrm{E}\left(G_{b 12}\right)$ with

$$
G_{b 12}=\operatorname{sgn}\left(V_{b 1}-V_{b 2}\right)\left\{\delta_{b}\left(V_{b 1}\right)-\delta_{b}\left(V_{b 2}\right)\right\}
$$

where $\delta_{b}(v)=\mathrm{E}\left\{Y^{(1)}-Y^{(-1)} \mid V_{b}=v\right\}$ and $V_{b 1}$ and $V_{b 2}$ are the values of $V_{b}$ for two independent patients. For a given augmentation $A=a(\boldsymbol{X}), \gamma_{b}$ can be estimated by

$$
\widehat{y}_{b}(a)=\frac{2}{n(n-1)} \sum_{i<j} \widehat{G}_{b i j}(a),
$$

where

$$
\widehat{G}_{b i j}(a)=2 \operatorname{sgn}\left(V_{b i}-V_{b j}\right)\left\{T_{i}\left(Y_{i}-A_{i}\right)-T_{j}\left(Y_{j}-A_{j}\right)\right\} .
$$

Let $\gamma_{c}$ and $\widehat{\gamma}_{c}(a)$ be defined analogously for $V_{c}$. Our comparison of the two markers is based on the difference $\gamma_{b}-\gamma_{c}$, which is consistently estimated by $\widehat{y}_{b}(a)-\widehat{y}_{c}(a)$. For any fixed $a$, it follows from standard asymptotic theory that $\sqrt{n}\left\{\widehat{\gamma}_{b}(a)-\widehat{y}_{c}(a)-\left(y_{b}-y_{c}\right)\right\}$ converges to a normal distribution with mean zero and variance

$$
\sigma_{b c}^{2}(a)=4\left[\operatorname{cov}\left\{\widehat{G}_{b 12}(a), \widehat{G}_{b 13}(a)\right\}+\operatorname{cov}\left\{\widehat{G}_{c 12}(a), \widehat{G}_{c 13}(a)\right\}-2 \operatorname{cov}\left\{\widehat{G}_{b 12}(a), \widehat{G}_{c 13}(a)\right\}\right] .
$$

A consistent estimator of $\sigma_{b c}^{2}(a)$ is given by

$$
\widehat{\sigma}_{b c}^{2}(a)=\frac{8}{n(n-1)(n-2)} \sum_{j \neq i \neq k, j<k}\left\{\widehat{G}_{b i j}(a) \widehat{G}_{b i k}(a)+\widehat{G}_{c i j}(a) \widehat{G}_{c i k}(a)-2 \widehat{G}_{b i j}(a) \widehat{G}_{c i k}(a)\right\}-4\left\{\widehat{y}_{b}(a)-\widehat{y}_{c}(a)\right\}^{2}
$$


In Appendix C, we show that $\sigma_{b c}^{2}(a)$ is minimized by the same $a_{\text {opt }}$ defined by eq. (4). As in Section 2.2, we can estimate $a_{\text {opt }}(\boldsymbol{X})$ by $\widehat{a}_{\text {opt }}(\boldsymbol{X})=m(\boldsymbol{X} ; \widehat{\boldsymbol{\alpha}})$, where $m(\boldsymbol{X} ; \boldsymbol{\alpha})$ is a working model for $\mathrm{E}(Y \mid \boldsymbol{X})$ and $\widehat{\boldsymbol{\alpha}}$ is an estimate of $\boldsymbol{\alpha}$. The asymptotic behavior of $\widehat{y}_{b}\left(\widehat{a}_{\text {opt }}\right)-\widehat{y}_{c}\left(\widehat{a}_{\text {opt }}\right)$ is similar to that of $\widehat{y}\left(\widehat{a}_{\text {opt }}\right)$ and can be described by the last paragraph of Section 2.2 with some obvious modifications.

\subsection{Combining multiple markers}

Suppose $\boldsymbol{V}$ is a sub-vector of $\boldsymbol{X}$ consisting of two or more treatment selection markers. A natural question is how to combine the markers in $\boldsymbol{V}$ into a single marker $S=s(\boldsymbol{V})$. We propose to combine $\boldsymbol{V}$ by maximizing $\gamma_{s}$, the $y$-value of $S$. To minimize new notation, we will continue to use the notation in Section 2.1 with $V$ replaced by $\boldsymbol{V}$. For instance, $\delta(\boldsymbol{V})$ is the conditional effect of $t=1$ versus $t=-1$ given $\boldsymbol{V}$. It is easy to see that $\gamma_{s}$ is maximized by any $s$ that preserves the ordering by $\delta$ :

$$
s\left(\boldsymbol{V}_{1}\right)>s\left(\boldsymbol{V}_{2}\right) \text { whenever } \delta\left(\boldsymbol{V}_{1}\right)>\delta\left(\boldsymbol{V}_{2}\right) .
$$

Thus, $\delta(\boldsymbol{V})$ itself would be an ideal choice of $S$, as would any strictly increasing function of $\delta(\boldsymbol{V})$.

The foregoing discussion suggests that we could set $S$ equal to an estimate of $\delta(\boldsymbol{V})$, which may be obtained under a model for $\mathrm{E}(Y \mid T, \boldsymbol{V})$ [24]. Noting that

$$
\mathrm{E}(Y \mid T, \boldsymbol{V})=\mu(\boldsymbol{V})+T \delta(\boldsymbol{V}) / 2,
$$

where $\mu(\boldsymbol{V})=\mathrm{E}\left\{Y^{(1)}+Y^{(-1)} \mid \boldsymbol{V}\right\} / 2$, we might parameterize $\mu(\boldsymbol{V})$ and $\delta(\boldsymbol{V})$ separately and estimate the model parameters by (iteratively reweighted) least squares. The optimality of this approach generally depends on the correctness of modeling assumptions, although there may be some robustness with respect to $\mu(\boldsymbol{V})[7,19]$. If $\delta(\boldsymbol{V})$ is misspecified, this approach may lead to a sub-optimal choice of $S$ whose interpretation is unclear.

In the rest of this subsection, we consider an alternative approach based on direct maximization of $\gamma_{s}$, similar in spirit to the approach of Zhang et al. [11] for estimating optimal treatment regimes. For practicality, we restrict attention to a parametric family of functions, $s(\boldsymbol{V} ; \boldsymbol{\beta})$, indexed by a finite-dimensional parameter $\boldsymbol{\beta}$. The specification of $s(\boldsymbol{V} ; \boldsymbol{\beta})$ may be informed by a parametric model for $\delta(\boldsymbol{V})$, and may also incorporate practical considerations such as simplicity and interpretability. The parameter space for $\boldsymbol{\beta}$ may need to be restricted so as to ensure that $s\left(\boldsymbol{V} ; \boldsymbol{\beta}_{1}\right)$ and $s\left(\boldsymbol{V} ; \boldsymbol{\beta}_{2}\right)$ are not strictly increasing functions of each other if $\boldsymbol{\beta}_{1} \neq \boldsymbol{\beta}_{2}$. For example, if $s(\boldsymbol{V} ; \boldsymbol{\beta})=\boldsymbol{\beta}^{\prime} \boldsymbol{V}$, we might restrict $\boldsymbol{\beta}$ to the unit sphere or, alternatively, fix the first element of $\boldsymbol{\beta}$ at 1. Once such a parametric family is defined, the problem is then to estimate $\boldsymbol{\beta}_{\text {opt }}=\operatorname{argmax}_{\boldsymbol{\beta}} \gamma(\boldsymbol{\beta})$, where we write $\gamma(\boldsymbol{\beta})=\gamma_{s(; ; \boldsymbol{\beta})}$ for convenience. The estimand $\boldsymbol{\beta}_{\text {opt }}$ can be interpreted as the true value of $\boldsymbol{\beta}$ in the following single index model:

$$
\delta(\boldsymbol{V})=\xi(s(\boldsymbol{V} ; \boldsymbol{\beta}))
$$

where $\xi$ is an unknown, strictly increasing function. Because $\xi$ is unspecified, this approach is more flexible than the one based on a parametric model for $\delta(\boldsymbol{V})$. Even if the single index model is misspecfied, $s\left(\cdot ; \boldsymbol{\beta}_{\text {opt }}\right)$ remains interpretable as a local maximizer of $\gamma_{s}$ (within the specified parametric family).

We propose to estimate $\boldsymbol{\beta}_{\text {opt }}$ by maximizing a consistent estimate of $\gamma(\boldsymbol{\beta})$, which may be obtained as in Section 2.2. Let $\widehat{\gamma}(\boldsymbol{\beta}, a)$ be defined by eqs (2) and (3) with $V$ replaced by $s(\boldsymbol{V} ; \boldsymbol{\beta})$. The objective function $\boldsymbol{\beta} \mapsto \widehat{\gamma}(\boldsymbol{\beta}, a)$ can be maximized using a grid search algorithm when the effective dimension of $\boldsymbol{\beta}$ is one or two. For a higher-dimensional $\boldsymbol{\beta}$, a grid search may be impractical, and the discreteness of the sign function in eq. (3) may create difficulties in applying a more efficient algorithm. To deal with this problem, we follow the approach of Ma and Huang [18] and apply a gradient descent algorithm to a smoothed version of $\widehat{y}(\boldsymbol{\beta}, a)$. Specifically, we replace the sign function with $\lambda_{n}=2 K\left(\cdot / \epsilon_{n}\right)-1$, where $K$ is a smooth, symmetric distribution 
function and $\left(\epsilon_{n}\right)$ is a sequence of positive numbers decreasing to 0 . This leads to the smoothed estimator

$$
\widetilde{\gamma}(\boldsymbol{\beta}, a)=\frac{2}{n(n-1)} \sum_{i<j} \widetilde{G}_{i j}(\boldsymbol{\beta}, a),
$$

where

$$
\widetilde{G}_{i j}(\boldsymbol{\beta}, a)=2 \lambda_{n}\left(s\left(\boldsymbol{V}_{i} ; \boldsymbol{\beta}\right)-s\left(\boldsymbol{V}_{j} ; \boldsymbol{\beta}\right)\right)\left\{T_{i}\left(Y_{i}-A_{i}\right)-T_{j}\left(Y_{j}-A_{j}\right)\right\} .
$$

It can be shown as in Ma and Huang [18, online supplement] that, under regularity conditions, $\widetilde{\gamma}(\boldsymbol{\beta}, a)$ is asymptotically equivalent to $\widehat{y}(\boldsymbol{\beta}, a)$; see Appendix D for details. Thus, the optimal choice of $a$ and the related discussion in Section 2.2 remain applicable to $\widetilde{\gamma}(\boldsymbol{\beta}, a)$. Empirical rules for choosing $\epsilon_{n}$ are given in Ma and Huang [18, Section 3.5].

Because of the asymptotic equivalence of $\widehat{\gamma}(\boldsymbol{\beta}, a)$ and $\widetilde{\gamma}(\boldsymbol{\beta}, a)$, we will for simplicity focus on the unsmoothed version in the rest of this subsection. Let $\widehat{\boldsymbol{\beta}}_{\text {opt }}$ be a maximizer of $\widehat{y}(\cdot, \widehat{a})$, where $\widehat{a}$ may be fixed or estimated. The arguments of Han [25] and Sherman [26] can be adapted to show that, under regularity conditions, $\widehat{\boldsymbol{\beta}}_{\text {opt }}$ is consistent for $\boldsymbol{\beta}_{\text {opt }}$ and (for a suitable parameterization) asymptotically normal. Of particular interest to us is the quantity $y\left(\widehat{\boldsymbol{\beta}}_{\text {opt }}\right)$, which measures the performance of the estimated optimal combination. Asymptotic inference on this quantity can be based on the fact that

$$
\sqrt{n}\left\{\widehat{y}\left(\widehat{\boldsymbol{\beta}}_{\mathrm{opt}}, \widehat{a}\right)-\mathrm{y}\left(\widehat{\boldsymbol{\beta}}_{\mathrm{opt}}\right)\right\}=\sqrt{n}\left\{\hat{\mathrm{y}}\left(\boldsymbol{\beta}_{\mathrm{opt}}, a_{\infty}\right)-\mathrm{y}\left(\boldsymbol{\beta}_{\mathrm{opt}}\right)\right\}+o_{p}(1),
$$

where $a_{\infty}$ is the probability limit of $\widehat{a}$ (a sketch proof is given in Appendix D).

In finite samples, a re-substitution bias can arise from the fact that $\widehat{\boldsymbol{\beta}}_{\mathrm{opt}}$ is chosen to maximize $\widehat{\gamma}(\cdot, \widehat{a})$ [1]. In general, the maximum of an estimated objective function is an over-estimator of the true objective function evaluated at the maximizer [20, 21]. The re-substitution bias can be removed using the following cross-validation procedure. We propose to partition the study cohort randomly into a specified number, say $K$, of subsamples that are roughly equal in size. For each $k \in\{1, \ldots, K\}$, we use the $k$ th subsample as a validation sample and combine the other subsamples into a training sample. From the training sample we obtain

$$
\widehat{\boldsymbol{\beta}}_{\mathrm{opt}}^{(-k)}=\operatorname{argmax}_{\boldsymbol{\beta}} \widehat{\boldsymbol{\gamma}}^{(-k)}\left(\boldsymbol{\beta}, \widehat{\boldsymbol{a}}^{(-k)}\right)
$$

using the exact same method for obtaining $\widehat{\boldsymbol{\beta}}_{\text {opt }}$. The superscript $(-k)$ in the preceding display emphasizes that each estimate is based on the training sample alone. Next, we evaluate the estimated combination $s\left(\cdot ; \widehat{\boldsymbol{\beta}}_{\mathrm{opt}}^{(-k)}\right)$ in the validation sample and obtain $\widehat{\gamma}^{(k)}\left(\widehat{\boldsymbol{\beta}}_{\mathrm{opt}}^{(-k)}, \widehat{a}^{(k)}\right)$, where the superscript $(k)$ indicates that the corresponding estimate is based on the validation sample alone. Because $\widehat{\boldsymbol{\beta}}_{\text {opt }}^{(-k)}$ and $\widehat{\gamma}^{(k)}\left(\cdot, \widehat{a}^{(k)}\right)$ are based on independent data, $\widehat{\gamma}^{(k)}\left(\widehat{\boldsymbol{\beta}}_{\text {opt }}^{(-k)}, \widehat{a}^{(k)}\right)$ is free of re-substitution bias. The final cross-validated estimator of $\mathrm{y}\left(\widehat{\boldsymbol{\beta}}_{\text {opt }}\right)$ is given by

$$
\widehat{\gamma}^{\mathrm{cv}}\left(\widehat{\boldsymbol{\beta}}_{\mathrm{opt}}\right)=\frac{1}{K} \sum_{k=1}^{K} \widehat{\gamma}^{(k)}\left(\widehat{\boldsymbol{\beta}}_{\mathrm{opt}}^{(-k)}, \widehat{a}^{(k)}\right) \text {. }
$$

Note that $\widehat{\gamma}^{(k)}\left(\widehat{\boldsymbol{\beta}}_{\text {opt }}^{(-k)}, \widehat{a}^{(k)}\right)$ is really an estimator of $\mathrm{y}\left(\widehat{\boldsymbol{\beta}}_{\text {opt }}^{(-k)}\right)$, which measures the utility of $s\left(\cdot ; \widehat{\boldsymbol{\beta}}_{\text {opt }}^{(-k)}\right)$ based on a training sample. Because $s\left(\cdot ; \widehat{\boldsymbol{\beta}}_{\text {opt }}\right)$ is based on a larger sample and is expected to perform better, $\widehat{\gamma}^{(k)}\left(\widehat{\boldsymbol{\beta}}_{\mathrm{opt}}^{(-k)}, \widehat{\boldsymbol{a}}^{(k)}\right)$ and hence $\widehat{\gamma}^{\mathrm{cv}}\left(\widehat{\boldsymbol{\beta}}_{\text {opt }}\right)$ may be biased downward for estimating $\gamma\left(\widehat{\boldsymbol{\beta}}_{\text {opt }}\right)$, especially for small $K$. The choice of $K$ thus represents a trade-off between bias and computational demand - a larger $K$ decreases the downward bias in $\widehat{\gamma}^{(k)}\left(\widehat{\boldsymbol{\beta}}_{\text {opt }}^{(-k)}, \widehat{a}^{(k)}\right)$ at the expense of more computation. Once $K$ is chosen, the size of a subsample is determined as roughly $n / K$. If this number is large enough for reliable estimation of $a_{\text {opt }}$, we can set $\widehat{a}^{(k)}$ to be an estimate of $a_{\text {opt }}$ based on the $k$ th subsample; otherwise we can substitute a fixed $a$ for $\widehat{a}^{(k)}$. 
Because the terms $\widehat{\gamma}^{(k)}\left(\widehat{\boldsymbol{\beta}}_{\text {opt }}^{(-k)}, \widehat{a}^{(k)}\right), k=1, \ldots, K$, are not independent of each other, it is not straightforward to derive the variance of the cross-validated estimator $\widehat{\gamma}^{\mathrm{cv}}\left(\widehat{\boldsymbol{\beta}}_{\text {opt }}\right)$. Nonetheless, inference on $y\left(\widehat{\boldsymbol{\beta}}_{\text {opt }}\right)$ can be based on nonparametric bootstrap standard errors and confidence intervals. A nonparametric bootstrap sample of the same size as the original sample can be created by sampling with replacement. A crossvalidated estimate of $y\left(\widehat{\boldsymbol{\beta}}_{\text {opt }}\right)$ can be obtained from each bootstrap sample, repeating all steps in optimization and cross-validation. This procedure can be repeated many times to produce a collection of bootstrap estimates, from which a bootstrap standard error can be obtained as a sample standard deviation and confidence limits as empirical quantiles.

\section{Numerical results}

\subsection{Analysis of HIV trial data}

The ECHO and THRIVE studies enrolled a combined total of 1,374 treatment-naive HIV-infected adults (694 ECHO; 680 THRIVE) in multiple countries. Main inclusion criteria included a screening viral load of at least 5,000 copies/ml and confirmed viral sensitivity to background N[t]RTIs. The study subjects were randomized $1: 1$ to rilpivirine $25 \mathrm{mg}$ once daily or efavirenz $600 \mathrm{mg}$ once daily, both in conjunction with a background $\mathrm{N}[\mathrm{t}] \mathrm{RTI}$ regimen which consisted of tenofovir disoproxil fumarate (TDF) and emtricitabine (FTC) in ECHO and was based on the investigator's choice of TDF/FTC, zidovudine/lamivudine (3TC), or abacavir/3TC in THRIVE. Randomization was stratified by screening viral load $\left(\leq 10^{5}, 10^{5}-5 \times 10^{5},>5 \times 10^{5} \mathrm{copies} / \mathrm{ml}\right)$ and background $\mathrm{N}[\mathrm{t}] \mathrm{RTI}$ regimen (in THRIVE). A double-dummy design was used to keep patients masked from the assigned treatment. Patients were followed for up to 96 weeks, and the primary endpoint was virologic response at week 48 . The observed response rates were $82.2 \%$ for rilpivirine and $79.8 \%$ for efavirenz.

Here we use the proposed methodology to evaluate, compare and combine the two markers (BVL and BCD4) for choosing between rilpivirine $(T=1)$ and efavirenz $(T=-1)$. For ease of handling, we work with standardized versions of $-\log (\mathrm{BVL})$ (minus because higher values of BVL indicate smaller benefits of rilpivirine versus efavirenz) and $\log (\mathrm{BCD} 4+1)$ (where one is added to handle zero counts). The standardization consists of subtracting by the mean and dividing by the standard deviation. We note that $y$ is invariant under a strictly increasing transformation. The $y$-values of the two markers are estimated with $A=\widehat{a}(\boldsymbol{X})$ equal to 0 (i.e., no augmentation) or an estimate of $\mathrm{E}(Y), \mathrm{E}(Y \mid \boldsymbol{V})$ or $\mathrm{E}(Y \mid \boldsymbol{X})$, where $\boldsymbol{V}$ consists of the two transformed markers and $\boldsymbol{X}$ consists of age, gender, race, body mass index, and the two markers. We estimate $\mathrm{E}(Y)$ with the sample mean of $Y$, and estimate $\mathrm{E}(Y \mid \boldsymbol{V})$ and $\mathrm{E}(Y \mid \boldsymbol{X})$ with logistic regression models that include the specified covariates as linear terms (in addition to an intercept). For brevity, we denote these augmentation terms by $\widehat{\mathrm{E}}(Y), \widehat{\mathrm{E}}(Y \mid \boldsymbol{V})$ and $\widehat{\mathrm{E}}(Y \mid \boldsymbol{X})$, respectively. Our analysis is restricted to a subset of 1,314 subjects with complete covariate and outcome data.

Table 1 shows the point estimates and standard errors for the $y$-values of the two markers as well as their difference. As one might expect, the standard errors do not increase when new information is added to the augmentation term. The reductions are quite large when $A$ changes from 0 to $\widehat{\mathrm{E}}(Y)$, and negligible when $A$ changes from $\widehat{\mathrm{E}}(Y \mid \boldsymbol{V})$ to $\widehat{\mathrm{E}}(Y \mid \boldsymbol{X})$, suggesting that the additional covariates in $\boldsymbol{X}$ (relative to $\boldsymbol{V}$ ) are of limited

Table 1: Marker comparison based on HIV trial data: point estimates and analytical standard errors for the $y$-values of BVL and $\mathrm{BCD} 4$ as well as their difference (BCD4 - BVL), obtained with different choices of $\widehat{a}$.

\begin{tabular}{|c|c|c|c|c|c|c|}
\hline \multirow[t]{2}{*}{$\widehat{a}(\boldsymbol{X})$} & \multicolumn{3}{|c|}{ Point estimate } & \multicolumn{3}{|c|}{ Standard error } \\
\hline & BVL & BCD4 & Diff. & BVL & BCD4 & Diff. \\
\hline 0 & 0.143 & 0.021 & -0.122 & 0.056 & 0.057 & 0.070 \\
\hline$\widehat{\mathrm{E}}(Y)$ & 0.045 & 0.084 & 0.039 & 0.026 & 0.026 & 0.028 \\
\hline$\widehat{\mathrm{E}}(Y \mid \boldsymbol{V})$ & 0.046 & 0.083 & 0.037 & 0.025 & 0.025 & 0.028 \\
\hline$\widehat{\mathrm{E}}(Y \mid X)$ & 0.043 & 0.082 & 0.039 & 0.025 & 0.025 & 0.028 \\
\hline
\end{tabular}


prognostic value. The point estimates based on $A=0$ are visibly different from those based on non-zero augmentation terms, possibly due to the large variability for $A=0$. The non-zero augmentation terms lead to very similar results, which together indicate that BCD4 has a higher $y$-value than BVL, although the difference is not statistically significant.

Next, we consider how to combine the two markers linearly, so $s(\boldsymbol{V} ; \boldsymbol{\beta})=\boldsymbol{\beta}^{\prime} \boldsymbol{V}$ with $\|\boldsymbol{\beta}\|=1$. Linear combinations are easy to use in practice, even though they may not attain the global optimum. Our approach is to directly maximize an estimate of $y$, which may be obtained using one of the four methods described earlier (with different augmentation terms). The maximization is done using a grid search algorithm based on the representation $\boldsymbol{\beta}=(\cos \phi, \sin \phi)^{\prime}, \phi \in[0,2 \pi)$. As a comparator, we also consider an alternative approach based on the logistic regression model

$$
\operatorname{logit} \mathrm{P}(Y=1 \mid T, \boldsymbol{X} ; \boldsymbol{\eta})=\eta_{1}+\boldsymbol{\eta}_{X}^{\prime} \boldsymbol{X}+T\left(\eta_{T}+\boldsymbol{\eta}_{T V}^{\prime} \boldsymbol{V}\right),
$$

where $\boldsymbol{\eta}=\left(\eta_{1}, \boldsymbol{\eta}_{X}^{\prime}, \eta_{T}, \boldsymbol{\eta}_{T V}^{\prime}\right)^{\prime}$ is a vector of unknown regression parameters. Fitting this logistic regression model produces a maximum likelihood estimate of $\boldsymbol{\eta}_{T V}$, which is then normalized to provide a candidate combination of the two markers. The $\gamma$-value of the candidate combination based on logistic regression is estimated with $A=\widehat{\mathrm{E}}(Y \mid \boldsymbol{X})$. A 5-fold cross-validation procedure is also used to estimate the $\gamma$-value for each candidate combination (from either approach), with $A=\widehat{\mathrm{E}}(Y \mid \boldsymbol{X})$ in the validation step. Bootstrap standard errors are obtained from 1,000 bootstrap samples.

Table 2 shows the results for marker combination. For the proposed approach, the cross-validated $y$-estimate tends to increase when new information is added to the augmentation term. Except in the noaugmentation case, the proposed approach produces higher cross-validated $y$-estimates than the logistic regression approach, and both approaches suggest that BCD4 should play a much larger role than BVL in the linear combination. In the no-augmentation case, cross-validation reduces the estimate of $y$ dramatically for the proposed approach.

\subsection{Example-based simulation}

We now evaluate the performance of the proposed methodology in a simulation study mimicking the ECHO and THRIVE trials. We work with the same subset of 1,314 subjects analyzed in Section 3.1 with their original marker values, and generate $T$ and $Y$ randomly according to the observed pattern in the data. Specifically, we let $\mathrm{P}(T=1 \mid \boldsymbol{V})=\mathrm{P}(T=-1 \mid \boldsymbol{V})=1 / 2$ and, for each $t \in\{1,-1\}$, set $\mathrm{P}(Y=1 \mid T=t, \boldsymbol{V})=\mathrm{P}\left(Y^{(t)}=1 \mid \boldsymbol{V}\right)$ equal to a nonparametric estimate based on tensor product splines. Because of the curse of dimensionality, this data generation mechanism does not involve the covariates in $\boldsymbol{X}$ that are not in $\boldsymbol{V}$, which therefore will not be included in the analysis. One thousand datasets are simulated independently and analyzed as in Section 3.1 (except that $\boldsymbol{X}=\boldsymbol{V}$ in this simulation study).

Table 2: Marker combination based on HIV trial data: point estimates and bootstrap standard errors for $\widehat{\boldsymbol{\beta}}_{\text {opt }}, \widehat{\gamma}\left(\widehat{\boldsymbol{\beta}}_{\text {opt }}, \widehat{a}\right)$ and $\widehat{\gamma}^{\mathrm{cv}}\left(\widehat{\boldsymbol{\beta}}_{\mathrm{opt}}\right)$, obtained with different choices of $\widehat{a}$ in the proposed approach.

\begin{tabular}{|c|c|c|c|c|c|c|c|c|}
\hline \multirow[t]{3}{*}{$\widehat{a}(\boldsymbol{X})$} & \multicolumn{4}{|c|}{ Point estimate } & \multicolumn{4}{|c|}{ Standard error } \\
\hline & \multicolumn{3}{|c|}{$\widehat{\boldsymbol{\beta}}_{\text {opt }}$} & & \multicolumn{2}{|r|}{$\widehat{\boldsymbol{\beta}}_{\mathrm{opt}}$} & \multirow[b]{2}{*}{$\widehat{\mathrm{y}}\left(\widehat{\boldsymbol{\beta}}_{\mathrm{opt}}, \widehat{a}\right)$} & \multirow[b]{2}{*}{$\widehat{\gamma}^{\mathrm{c} v}\left(\widehat{\boldsymbol{\beta}}_{\text {opt }}\right)$} \\
\hline & BVL & BCD4 & $\widehat{y}\left(\widehat{\boldsymbol{\beta}}_{\mathrm{opt}}, \widehat{a}\right)$ & $\widehat{\gamma}^{c v}\left(\widehat{\boldsymbol{\beta}}_{\text {opt }}\right)$ & BVL & BCD4 & & \\
\hline \multicolumn{9}{|c|}{ Logistic regression } \\
\hline$\widehat{\mathrm{E}}(Y \mid \boldsymbol{X})$ & 0.445 & 0.895 & 0.078 & 0.079 & 0.343 & 0.209 & 0.025 & 0.028 \\
\hline \multicolumn{9}{|c|}{ Proposed approach } \\
\hline 0 & 1.000 & 0.016 & 0.144 & 0.049 & 0.206 & 0.453 & 0.053 & 0.038 \\
\hline$\widehat{\mathrm{E}}(Y)$ & 0.124 & 0.992 & 0.085 & 0.080 & 0.284 & 0.194 & 0.025 & 0.030 \\
\hline$\widehat{\mathrm{E}}(Y \mid \boldsymbol{V})$ & 0.186 & 0.983 & 0.085 & 0.083 & 0.280 & 0.196 & 0.024 & 0.029 \\
\hline$\widehat{\mathrm{E}}(Y \mid \boldsymbol{X})$ & 0.124 & 0.992 & 0.084 & 0.086 & 0.260 & 0.192 & 0.024 & 0.029 \\
\hline
\end{tabular}


Table 3 shows the results for marker comparison: empirical bias, standard deviation, mean standard error and empirical coverage probability (of $95 \%$ Wald confidence intervals) for estimating the $y$-values of the two markers as well as their difference with different augmentation terms $(0, \widehat{E}(Y)$ and $\widehat{E}(Y \mid \boldsymbol{V})$ but not $\widehat{\mathrm{E}}(Y \mid \boldsymbol{X}))$. It is clear in Table 3 that the estimators are virtually unbiased and generally become more efficient with increasing information in the augmentation, although the improvement from $\widehat{E}(Y)$ to $\widehat{E}(Y \mid \boldsymbol{V})$ is quite modest (presumably due to low prognostic value of $\boldsymbol{V}$ ). In all cases, the standard errors approximate the empirical standard deviations well on average, and the resulting confidence intervals have close-to-nominal coverage.

The results for marker combination are presented in Table 4, where the proposed approach with the aforementioned augmentation terms is compared with a logistic regression approach based on the following model:

$$
\operatorname{logit} \mathrm{P}(Y=1 \mid T, \boldsymbol{V} ; \boldsymbol{\eta})=\eta_{1}+\boldsymbol{\eta}_{V}^{\prime} \boldsymbol{V}+T\left(\eta_{T}+\boldsymbol{\eta}_{T V}^{\prime} \boldsymbol{V}\right),
$$

where $\boldsymbol{\eta}=\left(\eta_{1}, \boldsymbol{\eta}_{V}^{\prime}, \eta_{T}, \boldsymbol{\eta}_{T V}^{\prime}\right)^{\prime}$. The proposed approach tends to perform better with increasing information in the augmentation, producing candidate combinations that are closer on average to the optimal combination with a higher mean of $\mathrm{y}\left(\widehat{\boldsymbol{\beta}}_{\text {opt }}\right)$. By the same measures, logistic regression outperforms the proposed approach with no augmentation but not with non-zero augmentations. For the proposed approach, $\widehat{\gamma}\left(\widehat{\boldsymbol{\beta}}_{\text {opt }}, \widehat{a}\right.$ ) (without crossvalidation) tends to over-estimate $y\left(\widehat{\boldsymbol{\beta}}_{\text {opt }}\right)$, especially in the no-augmentation case, and the re-substitution bias is effectively corrected by cross-validation. Although $\widehat{\gamma}^{\mathrm{cv}}\left(\widehat{\boldsymbol{\beta}}_{\text {opt }}\right)$ tends to under-estimate $\gamma\left(\widehat{\boldsymbol{\beta}}_{\text {opt }}\right)$, it is on average closer to $y\left(\widehat{\boldsymbol{\beta}}_{\text {opt }}\right)$ than $\widehat{\gamma}\left(\widehat{\boldsymbol{\beta}}_{\text {opt }}, \widehat{a}\right)$ is. It is also worth noting that, for the proposed approach, the variability in $\widehat{\boldsymbol{\beta}}_{\text {opt }}$ and $\mathrm{y}\left(\widehat{\boldsymbol{\beta}}_{\text {opt }}\right)$ tends to decrease with increasing information in the augmentation.

Table 3: Marker comparison in example-based simulation: empirical bias, standard deviation (SD), mean standard error (SE) and empirical coverage probability (CP) (of 95\% Wald confidence intervals) for estimating the $y$-values of BVL and BCD4 as well as their difference (BCD4 - BVL) with different choices of $\widehat{a}$. The true values are 0.046 for BVL, 0.081 for BCD4, and 0.035 for the difference.

\begin{tabular}{|c|c|c|c|c|c|c|c|c|c|c|c|c|}
\hline \multirow[t]{2}{*}{$\widehat{a}(X)$} & \multicolumn{4}{|c|}{ Bias } & \multicolumn{3}{|c|}{ SD } & \multicolumn{3}{|c|}{ SE } & \multicolumn{2}{|r|}{$C P$} \\
\hline & BVL & BCD4 & Diff. & BVL & BCD4 & Diff. & BVL & BCD4 & Diff. & BVL & BCD4 & Diff. \\
\hline 0 & -0.002 & -0.001 & 0.001 & 0.059 & 0.057 & 0.070 & 0.057 & 0.057 & 0.069 & 0.937 & 0.948 & 0.945 \\
\hline$\widehat{\mathrm{E}}(Y)$ & -0.001 & 0.000 & 0.001 & 0.026 & 0.025 & 0.028 & 0.026 & 0.026 & 0.029 & 0.954 & 0.953 & 0.951 \\
\hline$\widehat{\mathrm{E}}(Y \mid \boldsymbol{V})$ & -0.001 & -0.001 & 0.000 & 0.024 & 0.024 & 0.028 & 0.025 & 0.025 & 0.029 & 0.953 & 0.958 & 0.952 \\
\hline
\end{tabular}

Table 4: Marker combination in example-based simulation: empirical means and standard deviations of $\widehat{\boldsymbol{\beta}}_{\text {opt }}, y\left(\widehat{\boldsymbol{\beta}}_{\text {opt }}\right), \widehat{\gamma}\left(\widehat{\boldsymbol{\beta}}_{\text {opt }}, \widehat{a}\right)$ and $\widehat{\gamma}^{\mathrm{cv}}\left(\widehat{\boldsymbol{\beta}}_{\text {opt }}\right)$, obtained with different choices of $\widehat{a}$ in the proposed approach. The true optimum is given by $\boldsymbol{\beta}_{\text {opt }}=(0.062,0.998)^{\prime}$ with $\mathrm{y}\left(\boldsymbol{\beta}_{\mathrm{opt}}\right)=0.081$.

\begin{tabular}{|c|c|c|c|c|c|c|c|c|c|c|}
\hline \multirow[t]{3}{*}{$\widehat{a}(V)$} & \multicolumn{5}{|c|}{ Mean } & \multicolumn{5}{|c|}{ Standard deviation } \\
\hline & \multicolumn{2}{|c|}{$\widehat{\boldsymbol{\beta}}_{\text {opt }}$} & \multirow[b]{2}{*}{$y\left(\widehat{\boldsymbol{\beta}}_{\text {opt }}\right)$} & \multirow[b]{2}{*}{$\widehat{y}\left(\widehat{\boldsymbol{\beta}}_{\mathrm{opt}}, \widehat{a}\right)$} & \multirow[b]{2}{*}{$\widehat{y}^{c v}\left(\widehat{\boldsymbol{\beta}}_{\text {opt }}\right)$} & \multicolumn{2}{|c|}{$\widehat{\boldsymbol{\beta}}_{\mathrm{opt}}$} & \multirow[b]{2}{*}{$\mathrm{y}\left(\widehat{\boldsymbol{\beta}}_{\mathrm{opt}}\right)$} & \multirow[b]{2}{*}{$\widehat{y}\left(\widehat{\boldsymbol{\beta}}_{\mathrm{opt}}, \widehat{a}\right)$} & \multirow[b]{2}{*}{$\widehat{\gamma}^{\mathrm{cv}}\left(\widehat{\boldsymbol{\beta}}_{\mathrm{opt}}\right)$} \\
\hline & BVL & BCD4 & & & & BVL & BCD4 & & & \\
\hline \multicolumn{11}{|c|}{ Logistic regression } \\
\hline$\widehat{\mathrm{E}}(Y \mid \boldsymbol{V})$ & 0.443 & 0.827 & 0.070 & 0.079 & 0.069 & 0.228 & 0.334 & 0.008 & 0.024 & 0.028 \\
\hline \multicolumn{11}{|c|}{ Proposed approach } \\
\hline 0 & 0.258 & 0.702 & 0.063 & 0.112 & 0.056 & 0.537 & 0.519 & 0.033 & 0.046 & 0.042 \\
\hline$\widehat{\mathrm{E}}(Y)$ & 0.186 & 0.921 & 0.077 & 0.087 & 0.073 & 0.250 & 0.327 & 0.011 & 0.023 & 0.029 \\
\hline$\widehat{\mathrm{E}}(Y \mid \boldsymbol{V})$ & 0.166 & 0.931 & 0.077 & 0.087 & 0.072 & 0.266 & 0.332 & 0.011 & 0.023 & 0.029 \\
\hline
\end{tabular}


Table 5: Marker comparison in additional simulations: empirical bias, standard deviation (SD), mean standard error (SE) and empirical coverage probability (CP) (of $95 \%$ Wald confidence intervals) for estimating $\gamma_{1}, \gamma_{2}$ and $\gamma_{2}-\gamma_{1}$ with different choices of $\widehat{a}$.

\begin{tabular}{|c|c|c|c|c|c|c|c|c|c|c|c|c|}
\hline \multirow[t]{2}{*}{$\widehat{a}(X)$} & \multicolumn{5}{|c|}{ Bias } & \multicolumn{2}{|l|}{ SD } & \multicolumn{3}{|c|}{ SE } & \multicolumn{2}{|r|}{$\mathrm{CP}$} \\
\hline & $\gamma_{1}$ & $y_{2}$ & $y_{2}-y_{1}$ & $\gamma_{1}$ & $y_{2}$ & $y_{2}-y_{1}$ & $\mathrm{y}_{1}$ & $y_{2}$ & $\gamma_{2}-\gamma_{1}$ & $y_{1}$ & $\gamma_{2}$ & $y_{2}-y_{1}$ \\
\hline \multicolumn{13}{|c|}{$\xi=$ identity, $\boldsymbol{\beta}_{V}=(1,1)^{\prime}, \gamma_{1}=\gamma_{2} \approx 2.247$} \\
\hline 0 & 0.020 & 0.015 & -0.005 & 0.207 & 0.217 & 0.285 & 0.212 & 0.212 & 0.282 & 0.950 & 0.942 & 0.946 \\
\hline$\widehat{\mathrm{E}}(Y)$ & 0.015 & 0.011 & -0.005 & 0.206 & 0.217 & 0.285 & 0.212 & 0.212 & 0.282 & 0.957 & 0.945 & 0.944 \\
\hline$\widehat{\mathrm{E}}(Y \mid \boldsymbol{V})$ & 0.003 & -0.006 & -0.008 & 0.188 & 0.197 & 0.238 & 0.191 & 0.191 & 0.230 & 0.955 & 0.945 & 0.941 \\
\hline$\widehat{\mathrm{E}}(Y \mid \boldsymbol{X})$ & -0.008 & -0.014 & -0.006 & 0.160 & 0.165 & 0.182 & 0.161 & 0.161 & 0.179 & 0.954 & 0.947 & 0.948 \\
\hline \multicolumn{13}{|c|}{$\xi=$ identity, $\boldsymbol{\beta}_{V}=(1,2)^{\prime}, \gamma_{1} \approx 2.247, \gamma_{2} \approx 4.494$} \\
\hline 0 & 0.019 & 0.026 & 0.007 & 0.275 & 0.244 & 0.330 & 0.278 & 0.246 & 0.327 & 0.950 & 0.946 & 0.942 \\
\hline$\widehat{\mathrm{E}}(Y)$ & 0.015 & 0.016 & 0.002 & 0.275 & 0.244 & 0.331 & 0.277 & 0.246 & 0.327 & 0.949 & 0.947 & 0.941 \\
\hline$\widehat{\mathrm{E}}(Y \mid \boldsymbol{V})$ & 0.000 & -0.021 & -0.021 & 0.258 & 0.228 & 0.283 & 0.261 & 0.228 & 0.283 & 0.953 & 0.948 & 0.941 \\
\hline$\widehat{\mathrm{E}}(Y \mid \boldsymbol{X})$ & -0.004 & -0.027 & -0.023 & 0.236 & 0.209 & 0.248 & 0.239 & 0.204 & 0.244 & 0.955 & 0.950 & 0.939 \\
\hline \multicolumn{13}{|c|}{$\xi=$ expit, $\boldsymbol{\beta}_{V}=(1,1)^{\prime}, \gamma_{1}=y_{2} \approx 0.417$} \\
\hline 0 & -0.005 & -0.002 & 0.003 & 0.172 & 0.171 & 0.258 & 0.172 & 0.172 & 0.263 & 0.957 & 0.946 & 0.953 \\
\hline$\widehat{\mathrm{E}}(Y)$ & -0.006 & -0.003 & 0.003 & 0.172 & 0.171 & 0.258 & 0.172 & 0.172 & 0.263 & 0.956 & 0.947 & 0.954 \\
\hline$\widehat{\mathrm{E}}(Y \mid \boldsymbol{V})$ & -0.006 & -0.009 & -0.003 & 0.145 & 0.148 & 0.202 & 0.146 & 0.146 & 0.206 & 0.956 & 0.941 & 0.949 \\
\hline$\widehat{\mathrm{E}}(Y \mid \boldsymbol{X})$ & -0.004 & -0.007 & -0.003 & 0.103 & 0.106 & 0.144 & 0.104 & 0.104 & 0.146 & 0.945 & 0.940 & 0.940 \\
\hline \multicolumn{13}{|c|}{$\xi=$ expit, $\boldsymbol{\beta}_{V}=(1,2)^{\prime}, \gamma_{1} \approx 0.322, \gamma_{2} \approx 0.669$} \\
\hline 0 & -0.006 & -0.003 & 0.004 & 0.171 & 0.176 & 0.266 & 0.174 & 0.172 & 0.264 & 0.950 & 0.950 & 0.953 \\
\hline$\widehat{\mathrm{E}}(Y)$ & -0.007 & -0.004 & 0.003 & 0.171 & 0.177 & 0.265 & 0.174 & 0.172 & 0.264 & 0.949 & 0.947 & 0.953 \\
\hline$\widehat{\mathrm{E}}(Y \mid \boldsymbol{V})$ & -0.007 & -0.012 & -0.005 & 0.142 & 0.153 & 0.206 & 0.148 & 0.146 & 0.207 & 0.960 & 0.939 & 0.944 \\
\hline$\widehat{\mathrm{E}}(Y \mid \boldsymbol{X})$ & -0.003 & -0.008 & -0.005 & 0.107 & 0.103 & 0.143 & 0.107 & 0.104 & 0.148 & 0.946 & 0.954 & 0.953 \\
\hline \multicolumn{13}{|c|}{$\xi=\operatorname{sgn}, \boldsymbol{\beta}_{V}=(1,1)^{\prime}, \gamma_{1}=\gamma_{2} \approx 1.333$} \\
\hline 0 & -0.010 & 0.008 & 0.018 & 0.183 & 0.189 & 0.292 & 0.185 & 0.185 & 0.288 & 0.958 & 0.938 & 0.947 \\
\hline$\widehat{\mathrm{E}}(Y)$ & -0.012 & 0.006 & 0.018 & 0.183 & 0.189 & 0.292 & 0.185 & 0.185 & 0.288 & 0.960 & 0.941 & 0.946 \\
\hline$\widehat{\mathrm{E}}(Y \mid \boldsymbol{V})$ & -0.018 & -0.006 & 0.012 & 0.160 & 0.160 & 0.234 & 0.161 & 0.161 & 0.236 & 0.949 & 0.941 & 0.949 \\
\hline$\widehat{\mathrm{E}}(Y \mid \boldsymbol{X})$ & -0.016 & -0.009 & 0.008 & 0.121 & 0.126 & 0.185 & 0.124 & 0.124 & 0.187 & 0.955 & 0.951 & 0.947 \\
\hline \multicolumn{13}{|c|}{$\xi=\operatorname{sgn}, \boldsymbol{\beta}_{V}=(1,2)^{\prime}, \gamma_{1} \approx 0.818, \gamma_{2} \approx 1.745$} \\
\hline 0 & 0.002 & 0.000 & -0.003 & 0.196 & 0.184 & 0.295 & 0.194 & 0.176 & 0.285 & 0.948 & 0.932 & 0.940 \\
\hline$\widehat{\mathrm{E}}(Y)$ & 0.000 & -0.004 & -0.005 & 0.196 & 0.183 & 0.295 & 0.194 & 0.176 & 0.285 & 0.946 & 0.936 & 0.941 \\
\hline$\widehat{\mathrm{E}}(Y \mid \boldsymbol{V})$ & -0.001 & -0.015 & -0.014 & 0.171 & 0.152 & 0.238 & 0.171 & 0.150 & 0.234 & 0.950 & 0.944 & 0.946 \\
\hline$\widehat{\mathrm{E}}(Y \mid \boldsymbol{X})$ & -0.003 & -0.016 & -0.013 & 0.139 & 0.110 & 0.184 & 0.137 & 0.110 & 0.183 & 0.950 & 0.946 & 0.936 \\
\hline
\end{tabular}

\subsection{Additional simulations}

Here we report additional simulation experiments with a continuous outcome $Y$, two markers in $\boldsymbol{V}$, and a separate baseline variable $W$ such that $\boldsymbol{X}=\left(\boldsymbol{V}^{\prime}, W\right)^{\prime}$. The three components of $\boldsymbol{X}$ are independently distributed as standard normal. As before, treatment is randomized $1: 1$, so that $\mathrm{P}(T=1 \mid \boldsymbol{X})=\mathrm{P}(T=-1 \mid \boldsymbol{X})=1 / 2$. Given $(\boldsymbol{X}, T)$, we generate $Y$ from the following single index model:

$$
Y=\alpha_{1}+\boldsymbol{\alpha}_{X}^{\prime} \boldsymbol{X}+T \xi\left(\boldsymbol{\beta}_{V}^{\prime} \boldsymbol{V}\right)+\epsilon,
$$

where $\alpha_{1}=0, \boldsymbol{\alpha}_{X}=(0.5,-0.5,1)^{\prime}, \boldsymbol{\beta}_{V}=(1,1)^{\prime}$ or $(1,2)^{\prime}, \xi$ may be identity, expit (i.e., $\left.\xi(u)=1 /\{1+\exp (-u)\}\right)$ or sgn, and $\epsilon \sim N(0,1)$ independently of $(\boldsymbol{X}, T)$. Thus, all three components of $\boldsymbol{X}$ are prognostic, and both components of $\boldsymbol{V}$ are effect-modifying. The value of $\boldsymbol{\beta}_{V}$ determines the relative strength of the two markers, and the choice of $\xi$ has a direct impact on $\delta(\boldsymbol{V})$, which describes the heterogeneity of the treatment effect. One thousand samples of size $n=500$ are generated independently and analyzed for marker comparison and combination.

The results for marker comparison are presented in Table 5 , where $y_{j}(j=1,2)$ denotes the $y$-value for the $j$ th marker in $\boldsymbol{V}$. Here, $\boldsymbol{\beta}_{V}=(1,1)^{\prime}$ represents the null case $\left(\mathrm{y}_{1}=\mathrm{y}_{2}\right)$ and $\boldsymbol{\beta}_{V}=(1,2)^{\prime}$ represents the alternative 
case $\left(\gamma_{1}<\gamma_{2}\right)$. The $\gamma^{\prime}$ 's are estimated with the same choices of $A=\widehat{a}(\boldsymbol{X})$ as before $(0, \widehat{\mathrm{E}}(Y), \widehat{\mathrm{E}}(Y \mid \boldsymbol{V})$ and $\widehat{\mathrm{E}}(Y \mid \boldsymbol{X}))$, expect that $\mathrm{E}(Y \mid \boldsymbol{V})$ and $\mathrm{E}(Y \mid \boldsymbol{X})$ are now estimated under linear regression models with $\boldsymbol{V}$ and $\boldsymbol{X}$, respectively, as linear terms (in addition to an intercept). The results in Table 5 are consistent with those in Table 3 in the sense of small bias in point estimation and variance estimation as well as good coverage of confidence intervals. In addition, Table 5 demonstrates that the prognostic ability of $\boldsymbol{X}$ translates into improved precision when $A$ changes from $\widehat{\mathrm{E}}(Y)$ to $\widehat{\mathrm{E}}(Y \mid \boldsymbol{V})$ and further to $\widehat{\mathrm{E}}(Y \mid \boldsymbol{X})$. Note that there is no improvement from $A=0$ to $A=\widehat{\mathrm{E}}(Y)$ because $\mathrm{E}(Y)=0$ in this simulation study.

The results for marker combination are shown in Table 3.3, where the proposed approach is compared with a linear regression approach based on the following working model:

$$
\mathrm{E}(Y \mid T, \boldsymbol{X} ; \boldsymbol{\eta})=\eta_{1}+\boldsymbol{\eta}_{X}^{\prime} \boldsymbol{X}+T\left(\eta_{T}+\boldsymbol{\eta}_{T V}^{\prime} \boldsymbol{V}\right),
$$

where $\boldsymbol{\eta}=\left(\eta_{1}, \boldsymbol{\eta}_{X}^{\prime}, \eta_{T}, \boldsymbol{\eta}_{T V}^{\prime}\right)^{\prime}$ is a vector of unknown regression parameters. This model is correct if $\xi$ is identity and incorrect if $\xi$ is expit or sgn. The working model is fitted by ordinary least squares and the resulting estimate of $\boldsymbol{\eta}_{T V}$ is normalized to provide a candidate combination of the two markers. The $y$-value of the candidate combination based on linear regression is estimated with $A=\widehat{\mathrm{E}}(Y \mid \boldsymbol{X})$. A 5-fold cross-validation procedure is also used to estimate the $y$-value for each candidate combination (from either approach), with $A=\widehat{\mathrm{E}}(Y \mid \boldsymbol{X})$ in the validation step. In Table 3.3, the proposed approach generally performs better in estimating $\boldsymbol{\beta}_{\text {opt }}$, in the sense of decreasing variability and (for $\xi=$ expit) decreasing bias, when new information is added to the augmentation term. These trends correspond to decreasing variability and (for $\xi=$ expit) increasing mean of $y\left(\widehat{\boldsymbol{\beta}}_{\text {opt }}\right)$ (calculated through numerical integration). In all cases considered here, the linear regression approach performs similarly to the proposed approach with $A=\widehat{\mathrm{E}}(Y \mid \boldsymbol{X})$. This phenomenon is reminiscent of the observation that logistic regression and direct maximization of AUC often perform similarly for combining multiple diagnostic markers [17, 27]. Table 3.3 also illustrates that the re-substitution bias may or may not be a serious issue and that cross-validation could introduce a substantial downward bias in estimating $y\left(\widehat{\boldsymbol{\beta}}_{\text {opt }}\right)$.

In Appendix E, we examine the performance of the proposed methods at a smaller sample size $(n=100)$. The results for marker comparison indicate that estimation of the optimal augmentation can result in a small-sample bias in $\widehat{y}\left(\widehat{a}_{\text {opt }}\right)$ and the associated standard error, and that in some cases the proposed Wald confidence intervals can have sub-nominal coverage probabilities. For marker combination, the smaller sample size provides an opportunity to evaluate the bootstrap standard errors and the associated confidence intervals for $\gamma\left(\widehat{\boldsymbol{\beta}}_{\text {opt }}\right)$. The results show that the bootstrap standard errors (based on 200 bootstrap samples) perform well in most cases, although serious undercoverage could result from a substantial bias in the point estimate of $y\left(\widehat{\boldsymbol{\beta}}_{\text {opt }}\right)$. These findings suggest that extra caution should be taken when applying the methods to small samples and when making inference about $y\left(\widehat{\boldsymbol{\beta}}_{\text {opt }}\right)$.

\section{Discussion}

We have proposed a quantitative concordance measure, $\gamma$, for comparing and combining treatment selection markers without specifying a cutoff value. Not requiring a cutoff value is a desirable feature because in practice the choice of cutoff may depend on safety, cost and individual preference. In that sense, $y$ is similar to the AUC for evaluating and comparing diagnostic markers, and we have shown that $y$ is indeed related to the area under the selection impact curve. Like the AUC, $y$ is invariant to monotone transformations of marker values. Unlike the AUC, $y$ does not have a universal scale, which makes it difficult to make comparisons across different treatment selection problems. However, for a given patient population and a given pair of treatments, $y$ does have a fixed scale and can be used to compare different treatment selection markers.

For combining multiple treatment selection markers, our simulation results show that the proposed approach often performs similarly to a regression approach based on an appropriate model for $\mathrm{E}(Y \mid T, \boldsymbol{X})$, which is again analogous to the literature on diagnostic markers. The proposed approach does seem advantageous in the HIV trials that motivated this research. In other applications, a simulation study could be conducted to compare the two approaches. If, for a given application, neither approach clearly outperforms 
Table 6: Marker combination in additional simulations: empirical means and standard deviations of $\widehat{\boldsymbol{\beta}}_{\text {opt }}, \gamma\left(\widehat{\boldsymbol{\beta}}_{\text {opt }}\right), \widehat{\gamma}\left(\widehat{\boldsymbol{\beta}}_{\text {opt }}, \widehat{a}\right)$ and $\widehat{\gamma}^{\mathrm{cv}}\left(\widehat{\boldsymbol{\beta}}_{\text {opt }}\right)$, obtained with different choices of $\widehat{a}$ in the proposed approach.

\begin{tabular}{|c|c|c|c|c|c|c|c|c|c|c|}
\hline \multirow[t]{2}{*}{$\widehat{a}(X)$} & & \multicolumn{4}{|r|}{ Mean } & & & & \multicolumn{2}{|c|}{ Standard deviation } \\
\hline & & $\widehat{\boldsymbol{\beta}}_{\text {opt }}$ & $\mathrm{y}\left(\widehat{\boldsymbol{\beta}}_{\mathrm{opt}}\right)$ & $\widehat{y}\left(\widehat{\boldsymbol{\beta}}_{\mathrm{opt}}, \widehat{a}\right)$ & $\widehat{\gamma}^{\mathrm{cv}}\left(\widehat{\boldsymbol{\beta}}_{\mathrm{opt}}\right)$ & & $\widehat{\boldsymbol{\beta}}_{\text {opt }}$ & $\mathrm{y}\left(\widehat{\boldsymbol{\beta}}_{\text {opt }}\right)$ & $\widehat{y}\left(\widehat{\boldsymbol{\beta}}_{\mathrm{opt}}, \widehat{a}\right)$ & $\widehat{\gamma}^{\mathrm{cv}}\left(\widehat{\boldsymbol{\beta}}_{\mathrm{opt}}\right)$ \\
\hline \multicolumn{11}{|c|}{$\xi=$ identity, $\boldsymbol{\beta}_{V}=(1,1)^{\prime}, \boldsymbol{\beta}_{\text {opt }}=(0.707,0.707)^{\prime}, \mathrm{y}\left(\boldsymbol{\beta}_{\text {opt }}\right)=3.178$} \\
\hline$\widehat{\mathrm{E}}(Y \mid \boldsymbol{X})$ & 0.706 & 0.708 & 3.176 & 3.160 & 3.018 & 0.022 & 0.022 & 0.002 & 0.146 & 0.151 \\
\hline \multicolumn{11}{|c|}{ Proposed approach } \\
\hline 0 & 0.704 & 0.707 & 3.170 & 3.199 & 3.008 & 0.049 & 0.049 & 0.011 & 0.184 & 0.151 \\
\hline$\widehat{\mathrm{E}}(Y)$ & 0.704 & 0.707 & 3.170 & 3.193 & 3.009 & 0.049 & 0.049 & 0.011 & 0.183 & 0.152 \\
\hline$\widehat{\mathrm{E}}(Y \mid \boldsymbol{V})$ & 0.705 & 0.707 & 3.173 & 3.169 & 3.013 & 0.039 & 0.039 & 0.007 & 0.175 & 0.154 \\
\hline$\widehat{\mathrm{E}}(Y \mid \boldsymbol{X})$ & 0.705 & 0.708 & 3.175 & 3.160 & 3.014 & 0.029 & 0.029 & 0.004 & 0.146 & 0.155 \\
\hline \multicolumn{11}{|c|}{$\begin{array}{c}\xi=\text { identity, } \boldsymbol{\beta}_{V}=(1,2)^{\prime}, \boldsymbol{\beta}_{\mathrm{opt}}=(0.447,0.894)^{\prime}, \mathrm{y}\left(\boldsymbol{\beta}_{\mathrm{opt}}\right)=5.024 \\
\text { Linear regression }\end{array}$} \\
\hline$\widehat{\mathrm{E}}(Y \mid \boldsymbol{X})$ & 0.448 & 0.894 & 5.023 & 4.988 & 4.763 & 0.018 & 0.009 & 0.001 & 0.193 & 0.201 \\
\hline \multicolumn{11}{|c|}{ Proposed approach } \\
\hline 0 & 0.447 & 0.893 & 5.019 & 5.049 & 4.758 & 0.041 & 0.020 & 0.007 & 0.234 & 0.206 \\
\hline$\widehat{\mathrm{E}}(Y)$ & 0.448 & 0.893 & 5.019 & 5.039 & 4.754 & 0.040 & 0.020 & 0.007 & 0.235 & 0.206 \\
\hline$\widehat{\mathrm{E}}(Y \mid \boldsymbol{V})$ & 0.448 & 0.893 & 5.021 & 4.998 & 4.759 & 0.033 & 0.017 & 0.005 & 0.220 & 0.207 \\
\hline$\widehat{\mathrm{E}}(Y \mid \boldsymbol{X})$ & 0.448 & 0.893 & 5.022 & 4.988 & 4.765 & 0.026 & 0.014 & 0.003 & 0.193 & 0.205 \\
\hline \multicolumn{11}{|c|}{$\begin{array}{c}\xi=\text { expit, } \boldsymbol{\beta}_{V}=(1,1)^{\prime}, \boldsymbol{\beta}_{\mathrm{opt}}=(0.707,0.707)^{\prime}, \mathrm{y}\left(\boldsymbol{\beta}_{\mathrm{opt}}\right)=0.603 \\
\text { Linear regression }\end{array}$} \\
\hline \multicolumn{11}{|c|}{ Linear regression } \\
\hline$\widehat{\mathrm{E}}(Y \mid \boldsymbol{X})$ & 0.689 & 0.703 & 0.593 & 0.599 & 0.554 & 0.125 & 0.121 & 0.014 & 0.105 & 0.110 \\
\hline \multicolumn{11}{|c|}{ Proposed approach } \\
\hline 0 & 0.667 & 0.676 & 0.571 & 0.628 & 0.529 & 0.223 & 0.222 & 0.049 & 0.160 & 0.123 \\
\hline$\widehat{\mathrm{E}}(Y)$ & 0.667 & 0.675 & 0.570 & 0.627 & 0.526 & 0.223 & 0.223 & 0.049 & 0.160 & 0.121 \\
\hline$\widehat{\mathrm{E}}(Y \mid \boldsymbol{V})$ & 0.674 & 0.693 & 0.581 & 0.609 & 0.540 & 0.184 & 0.181 & 0.034 & 0.142 & 0.116 \\
\hline$\widehat{\mathrm{E}}(Y \mid \boldsymbol{X})$ & 0.692 & 0.700 & 0.593 & 0.601 & 0.554 & 0.127 & 0.124 & 0.014 & 0.105 & 0.110 \\
\hline \multicolumn{11}{|c|}{$\xi=$ expit, $\boldsymbol{\beta}_{V}=(1,2)^{\prime}, \boldsymbol{\beta}_{\mathrm{opt}}=(0.447,0.894)^{\prime}, \mathrm{\gamma}\left(\boldsymbol{\beta}_{\mathrm{opt}}\right)=0.758$} \\
\hline \multicolumn{11}{|c|}{ Linear regression } \\
\hline$\widehat{\mathrm{E}}(Y \mid \boldsymbol{X})$ & 0.457 & 0.878 & 0.749 & 0.753 & 0.708 & 0.126 & 0.068 & 0.014 & 0.104 & 0.108 \\
\hline \multicolumn{11}{|c|}{ Proposed approach } \\
\hline 0 & 0.452 & 0.858 & 0.732 & 0.781 & 0.686 & 0.211 & 0.124 & 0.040 & 0.159 & 0.116 \\
\hline$\widehat{\mathrm{E}}(Y)$ & 0.451 & 0.859 & 0.733 & 0.779 & 0.687 & 0.210 & 0.121 & 0.038 & 0.159 & 0.116 \\
\hline$\widehat{\mathrm{E}}(Y \mid \boldsymbol{V})$ & 0.445 & 0.875 & 0.742 & 0.766 & 0.697 & 0.168 & 0.090 & 0.023 & 0.142 & 0.111 \\
\hline$\widehat{\mathrm{E}}(Y \mid X)$ & 0.455 & 0.881 & 0.750 & 0.755 & 0.708 & 0.118 & 0.062 & 0.012 & 0.104 & 0.107 \\
\hline \multirow{2}{*}{\multicolumn{11}{|c|}{$\xi=\operatorname{sgn}, \boldsymbol{\beta}_{V}=(1,1)^{\prime}, \boldsymbol{\beta}_{\mathrm{opt}}=(0.707,0.707)^{\prime}, \mathrm{y}\left(\boldsymbol{\beta}_{\mathrm{opt}}\right)=2.000$}} \\
\hline \multicolumn{2}{|c|}{ Linear regression } & & & & & & & & & \\
\hline$\widehat{\mathrm{E}}(Y \mid \boldsymbol{X})$ & 0.706 & 0.705 & 1.995 & 1.983 & 1.902 & 0.047 & 0.047 & 0.007 & 0.107 & 0.110 \\
\hline \multicolumn{11}{|c|}{ Proposed approach } \\
\hline 0 & 0.700 & 0.709 & 1.990 & 2.011 & 1.895 & 0.062 & 0.062 & 0.014 & 0.162 & 0.111 \\
\hline$\widehat{\mathrm{E}}(Y)$ & 0.700 & 0.709 & 1.990 & 2.007 & 1.895 & 0.063 & 0.062 & 0.014 & 0.162 & 0.111 \\
\hline$\widehat{\mathrm{E}}(Y \mid \boldsymbol{V})$ & 0.703 & 0.707 & 1.994 & 1.988 & 1.899 & 0.050 & 0.050 & 0.009 & 0.145 & 0.112 \\
\hline$\widehat{\mathrm{E}}(\boldsymbol{Y} \mid \boldsymbol{X})$ & 0.705 & 0.707 & 1.996 & 1.985 & 1.903 & 0.038 & 0.038 & 0.005 & 0.106 & 0.110 \\
\hline \multicolumn{11}{|c|}{$\xi=\operatorname{sgn}, \boldsymbol{\beta}_{V}=(1,2)^{\prime}, \boldsymbol{\beta}_{\mathrm{opt}}=(0.447,0.894)^{\prime}, \mathrm{\gamma}\left(\boldsymbol{\beta}_{\mathrm{opt}}\right)=2.000$} \\
\hline \multicolumn{11}{|c|}{ Linear regression } \\
\hline$\widehat{\mathrm{E}}(Y \mid X)$ & 0.445 & 0.893 & 1.995 & 1.979 & 1.899 & 0.059 & 0.029 & 0.008 & 0.103 & 0.106 \\
\hline \multicolumn{11}{|c|}{ Proposed approach } \\
\hline 0 & 0.447 & 0.890 & 1.989 & 2.007 & 1.893 & 0.082 & 0.041 & 0.017 & 0.164 & 0.106 \\
\hline$\widehat{\mathrm{E}}(Y)$ & 0.447 & 0.890 & 1.989 & 2.003 & 1.891 & 0.083 & 0.041 & 0.017 & 0.164 & 0.108 \\
\hline$\widehat{\mathrm{E}}(Y \mid \boldsymbol{V})$ & 0.445 & 0.893 & 1.993 & 1.989 & 1.896 & 0.066 & 0.033 & 0.011 & 0.147 & 0.107 \\
\hline$\widehat{\mathrm{E}}(Y \mid \boldsymbol{X})$ & 0.446 & 0.893 & 1.996 & 1.981 & 1.900 & 0.049 & 0.024 & 0.006 & 0.102 & 0.106 \\
\hline
\end{tabular}


the other, both approaches could be included in a sensitivity analysis. Our simulation results also suggest that the re-substitution bias may or may not be a serious problem and that the cross-validated $y$-estimate for a candidate combination could be less or more biased (in the opposite direction) than the un-cross-validated estimate. These issues need to be investigated further, perhaps by means of simulation for a given application. Another area of future research is how to incorporate variable selection techniques such as the lasso into the proposed marker combination procedure when the dimension of $\boldsymbol{V}$ is very high (possibly greater than $n$ ).

Acknowledgment: We thank two anonymous reviewers for constructive comments that have improved the manuscript greatly. The views expressed in this article do not represent the official position of the U.S. Food and Drug Administration.

\section{References}

1. Simon R. Development and validation of biomarker classifiers for treatment selection. J Stat Plan Inference 2008;138:308-20.

2. Simon R. Clinical trials for predictive medicine: new challenges and paradigms. Clin Trials 2010;7:516-24.

3. Molina JM, Cahn P, Grinsztejn B, Lazzarin A, Mills A, Saag M. Rilpivirine versus efavirenz with tenofovir and emtricitabine in treatment-naive adults infected with HIV-1 (ECHO): a phase 3 randomised double-blind active-controlled trial. Lancet 2011;378:238-46.

4. Cohen CJ, Andrade-Villanueva J, Clotet B, Fourie J, Johnson MA, Ruxrungtham K. Rilpivirine versus efavirenz with two background nucleoside or nucleotide reverse transcriptase inhibitors in treatment-naive adults infected with HIV-1 (THRIVE): a phase 3, randomised, non-inferiority trial. Lancet 2011;378:229-37.

5. Cohen CJ, Molina JM, Cahn P, Clotet B, Fourie J, Grinsztejn B. Efficacy and safety of rilpivirine (TMC278) versus efavirenz at 48 weeks in treatment-naive HIV-1-infected patients: pooled results from the phase 3 double-blind randomized ECHO and THRIVE trials. J AIDS 2012;60:33-42.

6. Zhang Z, Nie L, Soon G, Liu A. The use of covariates and random effects in evaluating predictive biomarkers under a potential outcome framework. Ann Appl Stat 2014;8:2336-55.

7. Zhang Z, Qu Y, Zhang B, Nie L, Soon G. Use of auxiliary covariates in estimating a biomarker-adjusted treatment effect model with clinical trial data. Stat Methods Med Res 2016;25:2103-19.

8. Song X, Pepe MS. Evaluating markers for selecting a patients treatment. Biometrics 2004;60:874-83.

9. Qian M, Murphy SA. Performance guarantees for individualized treatment rules. Ann Stat 2011;39:1180-210.

10. Zhao Y, Zeng D, Rush J, Kosorok MR. Estimating individualized treatment rules using outcome weighted learning. J Am Stat Assoc 2012;107:1106-18.

11. Zhang B, Tsiatis A, Laber E, Davidian M. A robust method for estimating optimal treatment regimes. Biometrics 2012;68:1010-8.

12. Kang C, Janes H, Huang Y. Combining biomarkers to optimize patient treatment recommendations. Biometrics 2014;70:695707.

13. Janes H, Pepe MS, Bossuyt PM, Barlow WE. Measuring the performance of markers for guiding treatment decisions. Ann Intern Med 2011;154:253-9.

14. Zhou XH, Obuchowski NA, McClish DK. Statistical methods in diagnostic medicine. New York: Wiley, 2002.

15. Pepe MS. The statistical evaluation of medical tests for classification and prediction. New York: Oxford University Press, 2003.

16. McIntosh MS, Pepe MS. Combining several screening tests: optimality of the risk score. Biometrics 2002;58:657-64.

17. Pepe MS, Cai T, Longton G. Combining predictors for classification using the area under the receiver operating characteristic curve. Biometrics 2006;62;221-9.

18. Ma S, Huang J. Combining multiple markers for classification using ROC. Biometrics 2007;63:751-7.

19. Tian L, Alizadeh AA, Gentles AJ, Tibshirani R. A simple method for estimating interactions between a treatment and a large number of covariates. J Am Stat Assoc 2014;109:1517-32.

20. Dawid AP. Selection paradoxes of Bayesian inference. Multivariate Anal Appl 1994;24:211-20.

21. Senn S. A note concerning a selection "paradox" of Dawid's. Am Stat 2008;62:206-10.

22. Rubin DB. Estimating causal effects of treatments in randomized and nonrandomized studies. J Edu Psychol 1974;66:688-701.

23. van der Vaart AW. Asymptotic statistics. Cambridge: Cambridge University Press, 1998.

24. Cai T, Tian L, Wong PH, Wei LJ. Analysis of randomized comparative clinical trial data for personalized treatment selections. Biostatistics 2011;12:270-82.

25. Han AK. Non-parametric analysis of a generalized regression model. J Econ 1987;35:303-16.

26. Sherman RP. The limiting distribution of the maximum rank correlation estimator. Econometrica 1993;61:123-37.

27. Li KC, Duan N. Regression analysis under link violation. Ann Stat 1989;17:1009-52.

28. Nolan D, Pollard D. Functional limit theorems for $U$-processes. Ann Probab 1988;16:1291-8. 
29. van der Vaart AW, Wellner JA. Weak convergence and empirical processes with applications to statistics. New York: SpringerVerlag, 1996.

\section{Appendix A: Proof of (1)}

We start by noting that

$$
\begin{aligned}
\mathrm{y} & =\mathrm{E}\left\{\operatorname{sgn}\left(V_{1}-V_{2}\right) \delta\left(V_{1}\right)\right\}+\mathrm{E}\left\{\operatorname{sgn}\left(V_{2}-V_{1}\right) \delta\left(V_{2}\right)\right\} \\
& =2 \mathrm{E}\left\{\operatorname{sgn}\left(V_{1}-V_{2}\right) \delta\left(V_{1}\right)\right\} \\
& =2 \mathrm{E}\left[\mathrm{E}\left\{\operatorname{sgn}\left(V_{1}-V_{2}\right) \delta\left(V_{1}\right) \mid V_{1}\right\}\right] \\
& =2 \mathrm{E}\left\{\left(2 V_{1}-1\right) \delta\left(V_{1}\right)\right\} \\
& =4 \mathrm{E}\{V \delta(V)\}-2 \mathrm{E}\{\delta(V)\} \\
& =4 \mathrm{E}\{V \delta(V)\}-4 \mathrm{E}(V) \mathrm{E}\{\delta(V)\} \\
& =4 \operatorname{cov}\{V, \delta(V)\},
\end{aligned}
$$

which follows from the symmetry of $\left(V_{1}, V_{2}\right)$ and the assumed uniform distribution of $V$. Next, we note that

$$
\begin{aligned}
\theta(u) & =\int_{0}^{u} \mathrm{E}\left\{Y^{(-1)} \mid V=v\right\} \mathrm{d} v+\int_{u}^{1} \mathrm{E}\left\{Y^{(1)} \mid V=v\right\} \mathrm{d} v \\
& =\int_{0}^{1} \mathrm{E}\left\{Y^{(-1)} \mid V=v\right\} \mathrm{d} v-\int_{u}^{1} \mathrm{E}\left\{Y^{(-1)} \mid V=v\right\} \mathrm{d} v+\int_{u}^{1} \mathrm{E}\left\{Y^{(1)} \mid V=v\right\} \mathrm{d} v \\
& =\mathrm{E}\left\{Y^{(-1)}\right\}+\int_{u}^{1} \delta(v) \mathrm{d} v
\end{aligned}
$$

which implies that

$$
\begin{aligned}
\int_{0}^{1} \theta(u) \mathrm{d} u & =\mathrm{E}\left\{Y^{(-1)}\right\}+\int_{0}^{1} \int_{u}^{1} \delta(v) \mathrm{d} v \mathrm{~d} u \\
& =\mathrm{E}\left\{Y^{(-1)}\right\}+\int_{0}^{1} \int_{0}^{1} I(v>u) \delta(v) \mathrm{d} v \mathrm{~d} u \\
& =\mathrm{E}\left\{Y^{(-1)}\right\}+\int_{0}^{1} \int_{0}^{1} I(v>u) \delta(v) \mathrm{d} u \mathrm{~d} v \\
& =\mathrm{E}\left\{Y^{(-1)}\right\}+\int_{0}^{1} v \delta(v) \mathrm{d} v \\
& =\mathrm{E}\left\{Y^{(-1)}\right\}+\mathrm{E}\{V \delta(V)\}
\end{aligned}
$$

where $I(\cdot)$ is the indicator function. Combining eqs (6) with (7), we have

$$
\begin{aligned}
y & =4 \mathrm{E}\{V \delta(V)\}-4 \mathrm{E}(V) \mathrm{E}\{\delta(V)\} \\
& =4\left[\int_{0}^{1} \theta(u) \mathrm{d} u-\mathrm{E}\left\{Y^{(-1)}\right\}\right]-2 \Delta \\
& =4 \int_{0}^{1} \theta(u) \mathrm{d} u-4 \mathrm{E}\left\{Y^{(-1)}\right\}-2\left[\mathrm{E}\left\{Y^{(1)}\right\}-\mathrm{E}\left\{Y^{(-1)}\right\}\right] \\
& =4 \int_{0}^{1} \theta(u) \mathrm{d} u-2 \mathrm{E}\left\{Y^{(-1)}\right\}-2 \mathrm{E}\left\{Y^{(1)}\right\}
\end{aligned}
$$




\section{Appendix B: Theoretical Support for Section 2.2}

First, we note that, for two independent subjects $i \neq j$,

$$
\begin{aligned}
\mathrm{E}\left\{\widehat{G}_{i j}(a)\right\} & =\mathrm{E}\left[2 \operatorname{sgn}\left(V_{i}-V_{j}\right)\left\{T_{i}\left(Y_{i}-A_{i}\right)-T_{j}\left(Y_{j}-A_{j}\right)\right\}\right] \\
& =\mathrm{E}\left(\mathrm{E}\left[2 \operatorname{sgn}\left(V_{i}-V_{j}\right)\left\{T_{i}\left(Y_{i}-A_{i}\right)-T_{j}\left(Y_{j}-A_{j}\right)\right\} \mid \boldsymbol{V}_{i}, \boldsymbol{V}_{j}\right]\right) \\
& =\mathrm{E}\left(\operatorname{sgn}\left(V_{i}-V_{j}\right) \mathrm{E}\left[2\left\{T_{i}\left(Y_{i}-A_{i}\right)-T_{j}\left(Y_{j}-A_{j}\right)\right\} \mid \boldsymbol{V}_{i}, \boldsymbol{V}_{j}\right]\right) \\
& =\mathrm{E}\left(\operatorname{sgn}\left(V_{i}-V_{j}\right)\left[\mathrm{E}\left\{2 T_{i}\left(Y_{i}-A_{i}\right) \mid \boldsymbol{V}_{i}\right\}-\mathrm{E}\left\{2 T_{j}\left(Y_{j}-A_{j}\right) \mid \boldsymbol{V}_{j}\right\}\right]\right) \\
& =\mathrm{E}\left[\operatorname{sgn}\left(V_{i}-V_{j}\right)\left\{\delta\left(V_{i}\right)-\delta\left(V_{j}\right)\right\}\right] \\
& =\mathrm{E}\left\{G_{i j}\right\} \\
& =y,
\end{aligned}
$$

where we use the independence between subjects and the fact that $\mathrm{E}\{2 T(Y-A) \mid V\}=\delta(V)$ (noted in Section 2.2). Therefore, $\widehat{y}(a)$ is unbiased for $y$ for any fixed $a$.

For a fixed $a$, it follows from Theorem 12.3 of van der Vaart [23] that $\sqrt{n}\{\hat{y}(a)-y\}$ converges to a normal distribution with mean zero and variance

$$
\begin{aligned}
\sigma^{2}(a) & =4 \operatorname{cov}\left\{\widehat{G}_{12}(a), \widehat{G}_{13}(a)\right\} \\
& =4 \mathrm{E}\left\{\widehat{G}_{12}(a) \widehat{G}_{13}(a)\right\}-4 \mathrm{E}\left\{\widehat{G}_{12}(a)\right\} \mathrm{E}\left\{\widehat{G}_{13}(a)\right\} \\
& =4 \mathrm{E}\left\{\widehat{G}_{12}(a) \widehat{G}_{13}(a)\right\}-4 \mathrm{y}^{2} .
\end{aligned}
$$

A conditioning argument shows that

$$
\begin{aligned}
& \mathrm{E}\left\{\widehat{G}_{12}(a) \widehat{G}_{13}(a)\right\} \\
= & \mathrm{E}\left[4 \operatorname{sgn}\left(\left(V_{1}-V_{2}\right)\left(V_{1}-V_{3}\right)\right)\left\{T_{1}\left(Y_{1}-A_{1}\right)-T_{2}\left(Y_{2}-A_{2}\right)\right\}\left\{T_{1}\left(Y_{1}-A_{1}\right)-T_{3}\left(Y_{3}-A_{3}\right)\right\}\right] \\
= & \mathrm{E}\left(\mathrm { E } \left[4 \operatorname{sgn}\left(\left(V_{1}-V_{2}\right)\left(V_{1}-V_{3}\right)\right)\right.\right. \\
& \left.\left.\times\left\{T_{1}\left(Y_{1}-A_{1}\right)-T_{2}\left(Y_{2}-A_{2}\right)\right\}\left\{T_{1}\left(Y_{1}-A_{1}\right)-T_{3}\left(Y_{3}-A_{3}\right)\right\} \mid V_{1}, V_{2}, V_{3}\right]\right) \\
= & \mathrm{E}\left(\operatorname{sgn}\left(\left(V_{1}-V_{2}\right)\left(V_{1}-V_{3}\right)\right)\right. \\
& \left.\times \mathrm{E}\left[4\left\{T_{1}\left(Y_{1}-A_{1}\right)-T_{2}\left(Y_{2}-A_{2}\right)\right\}\left\{T_{1}\left(Y_{1}-A_{1}\right)-T_{3}\left(Y_{3}-A_{3}\right)\right\} \mid V_{1}, V_{2}, V_{3}\right]\right) \\
= & \mathrm{E}\left(\operatorname{sgn}\left(\left(V_{1}-V_{2}\right)\left(V_{1}-V_{3}\right)\right)\right. \\
& \times\left[\mathrm{E}\left\{4 T_{1}^{2}\left(Y_{1}-A_{1}\right)^{2} \mid V_{1}\right\}-\mathrm{E}\left\{4 T_{1}\left(Y_{1}-A_{1}\right) T_{2}\left(Y_{2}-A_{2}\right)\right) \mid V_{1}, V_{2}\right\} \\
& \left.\left.\left.\left.-\mathrm{E}\left\{4 T_{1}\left(Y_{1}-A_{1}\right) T_{3}\left(Y_{3}-A_{3}\right)\right) \mid V_{1}, V_{3}\right\}+\mathrm{E}\left\{4 T_{2}\left(Y_{2}-A_{2}\right) T_{3}\left(Y_{3}-A_{3}\right)\right) \mid V_{2}, V_{3}\right\}\right]\right) \\
= & \mathrm{E}\left(\operatorname{sgn}\left(\left(V_{1}-V_{2}\right)\left(V_{1}-V_{3}\right)\right)\right. \\
& \left.\times\left[\operatorname{var}\left\{2 T_{1}\left(Y_{1}-A_{1}\right) \mid V_{1}\right\}+\delta^{2}\left(V_{1}\right)-\delta\left(V_{1}\right) \delta\left(V_{2}\right)-\delta\left(V_{1}\right) \delta\left(V_{3}\right)+\delta\left(V_{2}\right) \delta\left(V_{3}\right)\right]\right) \\
= & \mathrm{E}\left(\operatorname{sgn}\left(\left(V_{1}-V_{2}\right)\left(V_{1}-V_{3}\right)\right)\left[\left\{\delta\left(V_{1}\right)-\delta\left(V_{2}\right)\right\}\left\{\delta\left(V_{1}\right)-\delta\left(V_{3}\right)\right\}+\operatorname{var}\left\{2 T_{1}\left(Y_{1}-A_{1}\right) \mid V_{1}\right\}\right]\right) \\
= & \mathrm{E}\left(G_{12} G_{13}\right)+\mathrm{E}\left[\operatorname{sgn}\left(\left(V_{1}-V_{2}\right)\left(V_{1}-V_{3}\right)\right) \operatorname{var}\left\{2 T_{1}\left(Y_{1}-A_{1}\right) \mid V_{1}\right\}\right] .
\end{aligned}
$$

We now consider minimizing $\sigma^{2}(a)$ with respect to $a$. Because $\mathrm{E}\left\{G_{12}(s) G_{13}(s)\right\}$ does not depend on $a$, we can focus on 


$$
\begin{aligned}
& \mathrm{E}\left[\operatorname{sgn}\left(\left(V_{1}-V_{2}\right)\left(V_{1}-V_{3}\right)\right) \operatorname{var}\left\{2 T_{1}\left(Y_{1}-A_{1}\right) \mid V_{1}\right\}\right] \\
= & \mathrm{E}\left(\mathrm{E}\left[\operatorname{sgn}\left(\left(V_{1}-V_{2}\right)\left(V_{1}-V_{3}\right)\right) \operatorname{var}\left\{2 T_{1}\left(Y_{1}-A_{1}\right) \mid V_{1}\right\} \mid V_{1}\right]\right) \\
= & \mathrm{E}\left\{\kappa\left(V_{1}\right) \tau^{2}\left(V_{1}\right)\right\},
\end{aligned}
$$

where

$$
\begin{aligned}
\kappa\left(V_{1}\right) & =\mathrm{E}\left\{\operatorname{sgn}\left(\left(V_{1}-V_{2}\right)\left(V_{1}-V_{3}\right)\right) \mid V_{1}\right\}, \\
\tau^{2}\left(V_{1}\right) & =\operatorname{var}\left\{2 T_{1}\left(Y_{1}-A_{1}\right) \mid V_{1}\right\} .
\end{aligned}
$$

Note that $\kappa\left(V_{1}\right)$ does not depend on $a$. Moreover, for any $v$,

$$
\kappa(v)=\{\mathrm{P}(V>v)-\mathrm{P}(V<v)\}^{2} \geq 0 .
$$

Thus, a choice of $a$ that minimizes $\tau^{2}(v)$ for every $v$ would minimize $\mathrm{E}\left\{\kappa\left(V_{1}\right) \tau^{2}\left(V_{1}\right)\right\}$ and hence $\sigma^{2}(a)$. Such a choice exists and is given by $a_{\mathrm{opt}}(\boldsymbol{X})=\mathrm{E}(Y \mid \boldsymbol{X})$. To see this, we write

$$
\begin{aligned}
\tau^{2}(v) & =\operatorname{var}\{2 T(Y-A) \mid V=v\} \\
& =\mathrm{E}[\operatorname{var}\{2 T(Y-A) \mid \boldsymbol{X}\} \mid V=v]+\operatorname{var}[\mathrm{E}\{2 T(Y-A) \mid \boldsymbol{X}\} \mid V=v],
\end{aligned}
$$

where

$$
\mathrm{E}\{2 T(Y-A) \mid \boldsymbol{X}\}=\mathrm{E}\left\{Y^{(1)}-Y^{(-1)} \mid \boldsymbol{X}\right\}=: d(\boldsymbol{X})
$$

does not depend on $A$, and

$$
\begin{aligned}
\operatorname{var}\{2 T(Y-A) \mid \boldsymbol{X}\} & =\mathrm{E}\left\{4 T^{2}(Y-A)^{2} \mid \boldsymbol{X}\right\}-\mathrm{E}\{2 T(Y-A) \mid \boldsymbol{X}\}^{2} \\
& =4 \mathrm{E}\left\{(Y-A)^{2} \mid \boldsymbol{X}\right\}-d^{2}(\boldsymbol{X})
\end{aligned}
$$

is minimized by $A=\mathrm{E}(Y \mid \boldsymbol{X})$.

Finally, let us consider $\widehat{\gamma}\left(\widehat{a}_{\text {opt }}\right)=\widehat{y}(m(\cdot ; \widehat{\boldsymbol{\alpha}}))$, where $\widehat{\boldsymbol{\alpha}}$ converges in probability to $\boldsymbol{\alpha}_{\infty}$. For any fixed $\boldsymbol{\alpha}$, it follows from the theory of U-statistics that

$$
\sqrt{n}\{\widehat{\gamma}(m(\cdot ; \boldsymbol{\alpha}))-\gamma\}=\frac{1}{\sqrt{n}} \sum_{i=1}^{n} h\left(\boldsymbol{X}_{i}, T_{i}, Y_{i} ; \boldsymbol{\alpha}\right)+o_{p}(1),
$$

where

$$
h(\boldsymbol{x}, t, y ; \boldsymbol{\alpha})=4 \mathrm{E}(\operatorname{sgn}(V-v)[T\{Y-m(\boldsymbol{X} ; \boldsymbol{\alpha})\}-t\{y-m(\boldsymbol{x} ; \boldsymbol{\alpha})\}])-2 \boldsymbol{y} .
$$

Under regularity conditions, we have

$$
\begin{aligned}
\sqrt{n}\{\widehat{\gamma}(m(\cdot ; \widehat{\boldsymbol{\alpha}}))-\mathrm{y}\}= & \frac{1}{\sqrt{n}} \sum_{i=1}^{n} h\left(\boldsymbol{X}_{i}, T_{i}, Y_{i} ; \widehat{\boldsymbol{\alpha}}\right)+o_{p}(1) \\
= & \frac{1}{\sqrt{n}} \sum_{i=1}^{n} h\left(\boldsymbol{X}_{i}, T_{i}, Y_{i} ; \boldsymbol{\alpha}_{\infty}\right) \\
& +\mathrm{E}\left\{\dot{\boldsymbol{h}}\left(\boldsymbol{X}, T, Y ; \boldsymbol{\alpha}_{\infty}\right)\right\} \sqrt{n}\left(\widehat{\boldsymbol{\alpha}}-\boldsymbol{\alpha}_{\infty}\right)+o_{p}(1),
\end{aligned}
$$


where

$$
\begin{aligned}
\dot{\boldsymbol{h}}(\boldsymbol{x}, t, y ; \boldsymbol{\alpha}) & =\partial h(\boldsymbol{x}, t, y ; \boldsymbol{\alpha}) / \partial \boldsymbol{\alpha} \\
& =-4 \mathrm{E}[\operatorname{sgn}(V-v)\{T \dot{\boldsymbol{m}}(\boldsymbol{X} ; \boldsymbol{\alpha})-\operatorname{t\dot {m}}(\boldsymbol{x} ; \boldsymbol{\alpha})\}],
\end{aligned}
$$

with $\dot{\boldsymbol{m}}(\boldsymbol{x} ; \boldsymbol{\alpha})=\partial m(\boldsymbol{x} ; \boldsymbol{\alpha}) / \partial \boldsymbol{\alpha}$. It is easy to see that

$$
\mathrm{E}\left\{\dot{\boldsymbol{h}}\left(\boldsymbol{X}, T, Y ; \boldsymbol{\alpha}_{\infty}\right)\right\}=-4 \mathrm{E}\left[\operatorname{sgn}\left(V_{1}-V_{2}\right)\left\{T_{1} \dot{\boldsymbol{m}}\left(\boldsymbol{X}_{1} ; \boldsymbol{\alpha}_{\infty}\right)-T_{2} \dot{\boldsymbol{m}}\left(\boldsymbol{X}_{2} ; \boldsymbol{\alpha}_{\infty}\right)\right\}\right]=\mathbf{0},
$$

because of the independence between randomized treatment and baseline covariates. Therefore, $\widehat{\gamma}\left(\widehat{a}_{\text {opt }}\right)$ is asymptotically equivalent to $\widehat{\gamma}\left(m\left(\cdot ; \boldsymbol{\alpha}_{\infty}\right)\right)$. If the model $m(\boldsymbol{X} ; \boldsymbol{\alpha})$ is correct, then $\boldsymbol{\alpha}_{\infty}$ is the true value of $\boldsymbol{\alpha}$ and $\widehat{y}\left(\widehat{a}_{\text {opt }}\right)$ is asymptotically equivalent to $\widehat{y}\left(a_{\text {opt }}\right)$.

\section{Appendix C: Theoretical Support for Section 2.3}

For any fixed $a$, the asymptotic variance of $\widehat{y}_{b}(a)-\widehat{y}_{c}(a)$ is given by

$$
\begin{aligned}
\sigma_{b c}^{2}(a) & =4\left[\operatorname{cov}\left\{\widehat{G}_{b 12}(a), \widehat{G}_{b 13}(a)\right\}+\operatorname{cov}\left\{\widehat{G}_{c 12}(a), \widehat{G}_{c 13}(a)\right\}-2 \operatorname{cov}\left\{\widehat{G}_{b 12}(a), \widehat{G}_{c 13}(a)\right\}\right] \\
& =4\left[\mathrm{E}\left\{\widehat{G}_{b 12}(a) \widehat{G}_{b 13}(a)\right\}+\mathrm{E}\left\{\widehat{G}_{c 12}(a) \widehat{G}_{c 13}(a)\right\}-2 \mathrm{E}\left\{\widehat{G}_{b 12}(a) \widehat{G}_{c 13}(a)\right\}\right]-4\left(\gamma_{b}-\gamma_{c}\right)^{2} .
\end{aligned}
$$

Write $\boldsymbol{V}=\left(V_{b}, V_{c}\right)$. It can be shown as in Appendix B that

$$
\mathrm{E}\left\{\widehat{G}_{b 12}(a) \widehat{G}_{b 13}(a)\right\}=\mathrm{E}\left(G_{b 12} G_{b 13}\right)+\mathrm{E}\left\{\kappa_{b}\left(\boldsymbol{V}_{1}\right) \tau^{2}\left(\boldsymbol{V}_{1}\right)\right\}
$$

where

$$
\begin{aligned}
& \kappa_{b}\left(\boldsymbol{V}_{1}\right)=\mathrm{E}\left\{\operatorname{sgn}\left(\left(V_{b 1}-V_{b 2}\right)\left(V_{b 1}-V_{b 3}\right)\right) \mid \boldsymbol{V}_{1}\right\}, \\
& \tau^{2}\left(\boldsymbol{V}_{1}\right)=\operatorname{var}\left\{2 T_{1}\left(Y_{1}-A_{1}\right) \mid \boldsymbol{V}_{1}\right\} .
\end{aligned}
$$

Note that we are now conditioning on $\boldsymbol{V}_{1}=\left(V_{b 1}, V_{c 1}\right)$ instead of $V_{b 1}$. Equation (8) remains true with the subscript $b$ replaced by $c$ and with $\kappa_{c}$ defined analogously. A similar argument shows that

$$
\mathrm{E}\left\{\widehat{G}_{b 12}(a) \widehat{G}_{c 13}(a)\right\}=\mathrm{E}\left(G_{b 12} G_{c 13}\right)+\mathrm{E}\left\{\kappa_{b c}\left(\boldsymbol{V}_{1}\right) \tau^{2}\left(\boldsymbol{V}_{1}\right)\right\}
$$

where

$$
\kappa_{b c}\left(\boldsymbol{V}_{1}\right)=\mathrm{E}\left\{\operatorname{sgn}\left(\left(V_{b 1}-V_{b 2}\right)\left(V_{c 1}-V_{c 3}\right)\right) \mid \boldsymbol{V}_{1}\right\} .
$$

Note that $\mathrm{E}\left(G_{b 12} G_{b 13}\right), \mathrm{E}\left(G_{c 12} G_{c 13}\right)$ and $\mathrm{E}\left(G_{b 12} G_{c 13}\right)$ do not depend on $a$. Therefore, for the purpose of minimizing $\sigma_{b c}^{2}(a)$ with respect to $a$, we can focus on minimizing

$$
\mathrm{E}\left[\left\{\kappa_{b}(\boldsymbol{V})+\kappa_{c}(\boldsymbol{V})-2 \kappa_{b c}(\boldsymbol{V})\right\} \tau^{2}(\boldsymbol{V})\right] .
$$

Here, the $\kappa$ 's do not depend on $a$. Moreover, for any $\boldsymbol{v}=\left(v_{b}, v_{c}\right)$,

$$
\kappa_{b}(\boldsymbol{v})+\kappa_{c}(\boldsymbol{v})-2 \kappa_{b c}(\boldsymbol{v})=\left\{\mathrm{P}\left(V_{b}>v_{b}\right)-\mathrm{P}\left(V_{b}<v_{b}\right)+\mathrm{P}\left(V_{c}>v_{c}\right)-\mathrm{P}\left(V_{c}<v_{c}\right)\right\}^{2} \geq 0 .
$$

Therefore, a choice of $a$ that minimizes $\tau^{2}(\boldsymbol{v})$ for every $\boldsymbol{v}$ would minimize $\sigma_{b c}^{2}(a)$. Such a choice exists and is given by $a_{\text {opt }}(\boldsymbol{X})=\mathrm{E}(Y \mid \boldsymbol{X})$, as we have shown in Appendix B. 
Let $\boldsymbol{\alpha}_{\infty}$ denote the probability limit of $\widehat{\boldsymbol{\alpha}}$. By applying the last paragraph of Appendix B to $V_{b}$ and $V_{c}$ separately, we obtain

$$
\begin{aligned}
& \sqrt{n}\left\{\widehat{y}_{b}\left(\widehat{a}_{\text {opt }}\right)-\gamma_{b}\right\}=\sqrt{n}\left\{\widehat{y}_{b}\left(m\left(\cdot ; \boldsymbol{\alpha}_{\infty}\right)\right)-\gamma_{b}\right\}+o_{p}(1), \\
& \sqrt{n}\left\{\widehat{y}_{c}\left(\widehat{a}_{\text {opt }}\right)-\gamma_{c}\right\}=\sqrt{n}\left\{\widehat{y}_{c}\left(m\left(\cdot ; \boldsymbol{\alpha}_{\infty}\right)\right)-\gamma_{c}\right\}+o_{p}(1),
\end{aligned}
$$

and hence

$$
\sqrt{n}\left\{\widehat{y}_{b}\left(\widehat{a}_{\mathrm{opt}}\right)-\widehat{\mathrm{y}}_{c}\left(\widehat{a}_{\mathrm{opt}}\right)-\left(\mathrm{y}_{b}-\mathrm{y}_{c}\right)\right\}=\sqrt{n}\left\{\widehat{\mathrm{y}}_{b}\left(m\left(\cdot ; \boldsymbol{\alpha}_{\infty}\right)\right)-\widehat{\mathrm{y}}_{c}\left(m\left(\cdot ; \boldsymbol{\alpha}_{\infty}\right)\right)-\left(\mathrm{y}_{b}-\mathrm{y}_{c}\right)\right\}+o_{p}(1)
$$

This shows that $\widehat{y}_{b}\left(\widehat{a}_{\text {opt }}\right)-\widehat{y}_{c}\left(\widehat{a}_{\text {opt }}\right)$ is asymptotically equivalent to $\widehat{y}_{b}\left(m\left(\cdot ; \boldsymbol{\alpha}_{\infty}\right)\right)-\widehat{y}_{c}\left(m\left(\cdot ; \boldsymbol{\alpha}_{\infty}\right)\right)$.

\section{Appendix D: Theoretical Support for Section 2.4}

First, we demonstrate the asymptotical equivalence of $\widetilde{\gamma}(\boldsymbol{\beta}, a)$ to $\widehat{y}(\boldsymbol{\beta}, a)$ by showing that

$$
\sqrt{n}\{\tilde{\gamma}(\boldsymbol{\beta}, a)-\widehat{\gamma}(\boldsymbol{\beta}, a)\}=o_{p}(1) .
$$

To fix ideas, we work with a specific kernel function, $K(u)=1 /\{1+\exp (-u)\}$, although the argument extends easily to other kernel functions. We also assume for simplicity that $|Y-A| \leq L<\infty$ with probability 1 , and write $S=s(\boldsymbol{V} ; \boldsymbol{\beta})$. For any $\delta_{n}>0$, we have

$$
\begin{aligned}
& |\widetilde{\gamma}(\boldsymbol{\beta}, a)-\widehat{\gamma}(\boldsymbol{\beta}, a)| \\
\leq & \left|\frac{1}{n(n-1)} \sum_{i \neq j} I\left(\left|S_{i}-S_{j}\right| \geq \delta_{n}\right)\left\{\lambda_{n}\left(S_{i}-S_{j}\right)-\operatorname{sgn}\left(S_{i}-S_{j}\right)\right\}\left\{T_{i}\left(Y_{i}-A_{i}\right)-T_{j}\left(Y_{j}-A_{j}\right)\right\}\right| \\
& +\left|\frac{1}{n(n-1)} \sum_{i \neq j} I\left(\left|S_{i}-S_{j}\right|<\delta_{n}\right)\left\{\lambda_{n}\left(S_{i}-S_{j}\right)-\operatorname{sgn}\left(S_{i}-S_{j}\right)\right\}\left\{T_{i}\left(Y_{i}-A_{i}\right)-T_{j}\left(Y_{j}-A_{j}\right)\right\}\right| \\
\leq & \frac{2 L}{n(n-1)} \sum_{i \neq j} I\left(\left|S_{i}-S_{j}\right| \geq \delta_{n}\right)\left|\lambda_{n}\left(S_{i}-S_{j}\right)-\operatorname{sgn}\left(S_{i}-S_{j}\right)\right|+\frac{4 L}{n(n-1)} \sum_{i \neq j} I\left(\left|S_{i}-S_{j}\right|<\delta_{n}\right) \\
= & : D_{n 1}+D_{n 2} .
\end{aligned}
$$

For $|u| \geq \delta_{n}$, we have $\left|\lambda_{n}(u)-\operatorname{sgn}(u)\right|<2 \exp \left(-|u| / \epsilon_{n}\right)<2 \exp \left(-\delta_{n} / \epsilon_{n}\right)$, so that

$$
\sqrt{n} D_{n 1} \leq 4 L \exp \left(2^{-1} \log n-\delta_{n} / \epsilon_{n}\right)
$$

It follows from Nolan and Pollard [28, proof of Theorem 5] that

$$
\sup _{\delta} \sqrt{n}\left|\frac{\sum_{i \neq j}\left\{I\left(\left|S_{i}-S_{j}\right|<\delta\right)-\mathrm{P}\left(\left|S_{1}-S_{2}\right|<\delta\right)\right\}}{n(n-1)}-\frac{2 \sum_{i=1}^{n}\left\{F_{-}\left(S_{i}+\delta\right)-F\left(S_{i}-\delta\right)-\mathrm{P}\left(\left|S_{1}-S_{2}\right|<\delta\right)\right\}}{n}\right|=o_{p}(1),
$$

where $F$ is the distribution function of $S$ and $F_{-}$is the left-hand limit of $F$. This implies that

$$
\begin{aligned}
& \sqrt{n}\left\{D_{n 2}-4 L \mathrm{P}\left(\left|S_{1}-S_{2}\right|<\delta_{n}\right)\right\} \\
= & \frac{8 L}{\sqrt{n}} \sum_{i=1}^{n}\left\{F_{-}\left(S_{i}+\delta_{n}\right)-F\left(S_{i}-\delta_{n}\right)-\mathrm{P}\left(\left|S_{1}-S_{2}\right|<\delta_{n}\right)\right\}+o_{p}(1) \\
= & o_{p}(1),
\end{aligned}
$$


where the second step follows from van der Vaart [23, Theorem 19.24]. If $S_{1}-S_{2}$ is continuously distributed in a neighborhood of 0 with continuous density, then $\sqrt{n} \mathrm{P}\left(\left|S_{1}-S_{2}\right|<\delta_{n}\right) \rightarrow 0$ if we set $\delta_{n}=n^{d_{2}}$ for some $d_{2}<-1 / 2$. For this choice of $\delta_{n}$, eq. (12) implies that $\sqrt{n} D_{n 2}=o_{p}$ (1). With $\epsilon_{n}=n^{d_{1}}$ for some $d_{1}<d_{2}$, eq. (11) implies that $\sqrt{n} D_{n 1}=o_{p}$ (1). Substituting these results into eq. (10) completes the proof of eq. (9).

Next, we give a sketch proof of eq. (5). It follows from Appendix B that, for any fixed $\boldsymbol{\beta}$,

$$
\sqrt{n}\{\widehat{\gamma}(\boldsymbol{\beta}, \widehat{a})-\gamma(\boldsymbol{\beta})\}=\frac{1}{\sqrt{n}} \sum_{i=1}^{n} h\left(\boldsymbol{X}_{i}, T_{i}, Y_{i} ; \boldsymbol{\beta}, a_{\infty}\right)+o_{p}(1),
$$

where

$$
h(\boldsymbol{x}, t, y ; \boldsymbol{\beta}, a)=4 \mathrm{E}(\operatorname{sgn}(s(\boldsymbol{V} ; \boldsymbol{\beta})-s(\boldsymbol{v} ; \boldsymbol{\beta}))[T\{Y-a(\boldsymbol{X})\}-t\{y-a(\boldsymbol{x})\}])-2 \mathrm{y}(\boldsymbol{\beta}) .
$$

Under regularity conditions, we have

$$
\begin{aligned}
\sqrt{n}\left\{\widehat{\mathrm{y}}\left(\widehat{\boldsymbol{\beta}}_{\mathrm{opt}}, \widehat{a}\right)-\mathrm{y}\left(\widehat{\boldsymbol{\beta}}_{\mathrm{opt}}\right)\right\} & =\frac{1}{\sqrt{n}} \sum_{i=1}^{n} h\left(\boldsymbol{X}_{i}, T_{i}, Y_{i} ; \widehat{\boldsymbol{\beta}}_{\mathrm{opt}}, a_{\infty}\right)+o_{p}(1) \\
& =\frac{1}{\sqrt{n}} \sum_{i=1}^{n} h\left(\boldsymbol{X}_{i}, T_{i}, Y_{i} ; \boldsymbol{\beta}_{\mathrm{opt}}, a_{\infty}\right)+o_{p}(1) \\
& =\sqrt{n}\left\{\widehat{\gamma}\left(\boldsymbol{\beta}_{\mathrm{opt}}, \widehat{a}\right)-\mathrm{y}\left(\boldsymbol{\beta}_{\mathrm{opt}}\right)\right\}+o_{p}(1),
\end{aligned}
$$

where the second step can be established using Theorem 19.24 of van der Vaart [23], assuming that

$$
\left\{h\left(\boldsymbol{X}, T, Y ; \boldsymbol{\beta}, a_{\infty}\right):\left\|\boldsymbol{\beta}-\boldsymbol{\beta}_{\text {opt }}\right\|<\epsilon\right\}
$$

is a Donsker class [29] for some $\epsilon>0$ and that

$$
\left\|h\left(\boldsymbol{X}, T, Y ; \widehat{\boldsymbol{\beta}}_{\mathrm{opt}}, a_{\infty}\right)-h\left(\boldsymbol{X}, T, Y ; \boldsymbol{\beta}_{\mathrm{opt}}, a_{\infty}\right)\right\|_{2, P}=o_{p}(1) .
$$

Incidentally, eq. (5) remains valid with $y\left(\widehat{\boldsymbol{\beta}}_{\text {opt }}\right)$ replaced by $\mathrm{y}\left(\boldsymbol{\beta}_{\text {opt }}\right)$, because

$$
\begin{aligned}
& \sqrt{n}\left\{\widehat{y}\left(\widehat{\boldsymbol{\beta}}_{\mathrm{opt}}, \widehat{a}\right)-\mathrm{y}\left(\boldsymbol{\beta}_{\mathrm{opt}}\right)\right\} \\
= & \sqrt{n}\left\{\hat{\mathrm{y}}\left(\widehat{\boldsymbol{\beta}}_{\mathrm{opt}}, \widehat{a}\right)-\mathrm{y}\left(\widehat{\boldsymbol{\beta}}_{\mathrm{opt}}\right)\right\}+\sqrt{n}\left\{\mathrm{y}\left(\widehat{\boldsymbol{\beta}}_{\mathrm{opt}}\right)-\mathrm{y}\left(\boldsymbol{\beta}_{\mathrm{opt}}\right)\right\} \\
= & \frac{1}{\sqrt{n}} \sum_{i=1}^{n} h\left(\boldsymbol{X}_{i}, T_{i}, Y_{i} ; \boldsymbol{\beta}_{\mathrm{opt}}, a_{\infty}\right)+\sqrt{n}\left(\widehat{\boldsymbol{\beta}}_{\mathrm{opt}}-\boldsymbol{\beta}_{\mathrm{opt}}\right)^{\prime} \partial \mathrm{y}\left(\boldsymbol{\beta}_{\mathrm{opt}}\right) / \partial \boldsymbol{\beta}+o_{p}(1) \\
= & \frac{1}{\sqrt{n}} \sum_{i=1}^{n} h\left(\boldsymbol{X}_{i}, T_{i}, Y_{i} ; \boldsymbol{\beta}_{\mathrm{opt}}, a_{\infty}\right)+o_{p}(1)
\end{aligned}
$$

where the last step follows from the fact that $\boldsymbol{\beta}_{\text {opt }}$ maximizes $\mathrm{y}(\boldsymbol{\beta})$, which implies that $\partial \mathrm{y}\left(\boldsymbol{\beta}_{\text {opt }}\right) / \partial \boldsymbol{\beta}=\mathbf{0}$. A similar argument has been made by Zhang et al. [11] in the context of estimating optimal treatment regimes. This result implies that an asymptotic confidence interval for $\gamma\left(\widehat{\boldsymbol{\beta}}_{\text {opt }}\right)$ based on eq. (5) is also an asymptotic confidence interval for $\mathrm{y}\left(\boldsymbol{\beta}_{\text {opt }}\right)$.

\section{Appendix E: Simulation Results for $n=100$}

Here we report further simulation results obtained in the situation of Section 3.3 with $n=100$. Table 7 presents the results for marker combination in the same format as Table 5. As expected, the methods generally have 
inferior performance at $n=100$ as compared to $n=500$, especially when a regression model is fitted to estimate $\mathrm{E}(Y \mid \boldsymbol{V})$ or $\mathrm{E}(Y \mid \boldsymbol{X})$. Specifically, the point estimates are visibly biased, the standard errors tend to under-estimate the true standard deviations, and the confidence intervals have sub-nominal coverage probabilities in some cases. Table 8 shows the results for marker combination in a similar format to Table 3.3. In terms of $\mathrm{y}\left(\widehat{\boldsymbol{\beta}}_{\mathrm{opt}}\right)$, the proposed approach generally performs better with increasing information in $A$, and the linear regression approach performs similarly to the proposed approach with $A=\widehat{E}(Y \mid \boldsymbol{X})$. As in Table 3.3, the re-substitution bias may or may not be a serious issue, and cross-validation could introduce a substantial downward bias in estimating $y\left(\widehat{\boldsymbol{\beta}}_{\text {opt }}\right)$. The smaller sample size provides an opportunity to evaluate the bootstrap standard errors and the associated confidence intervals for $y\left(\widehat{\boldsymbol{\beta}}_{\text {opt }}\right)$. The corresponding results in Table 8 show that the bootstrap standard errors (based on 200 bootstrap samples) perform well in most cases, although serious undercoverage (say, less than 92\%) could result from a substantial bias in the point estimate of $\mathrm{y}\left(\widehat{\boldsymbol{\beta}}_{\text {opt }}\right)$. 


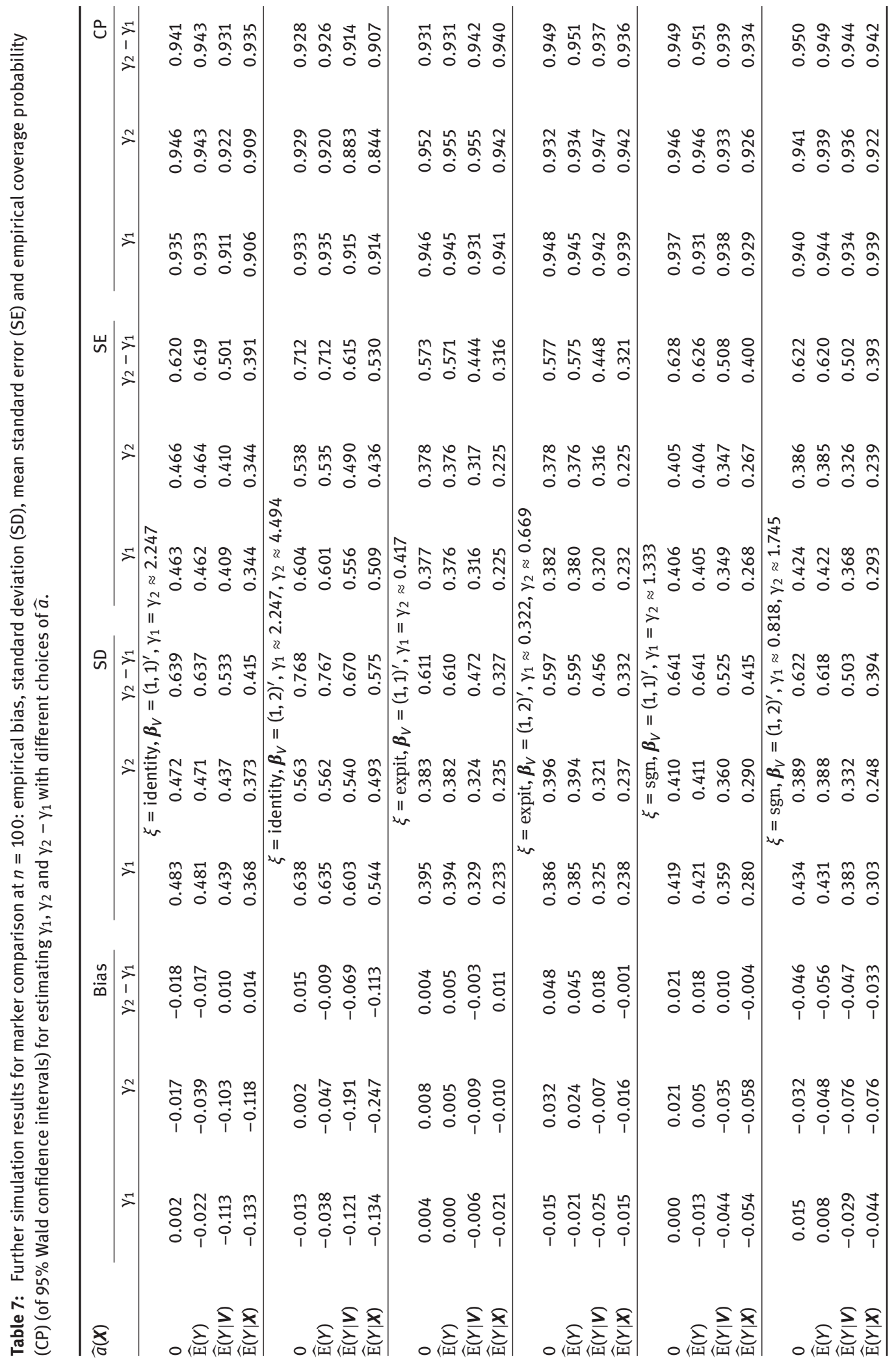




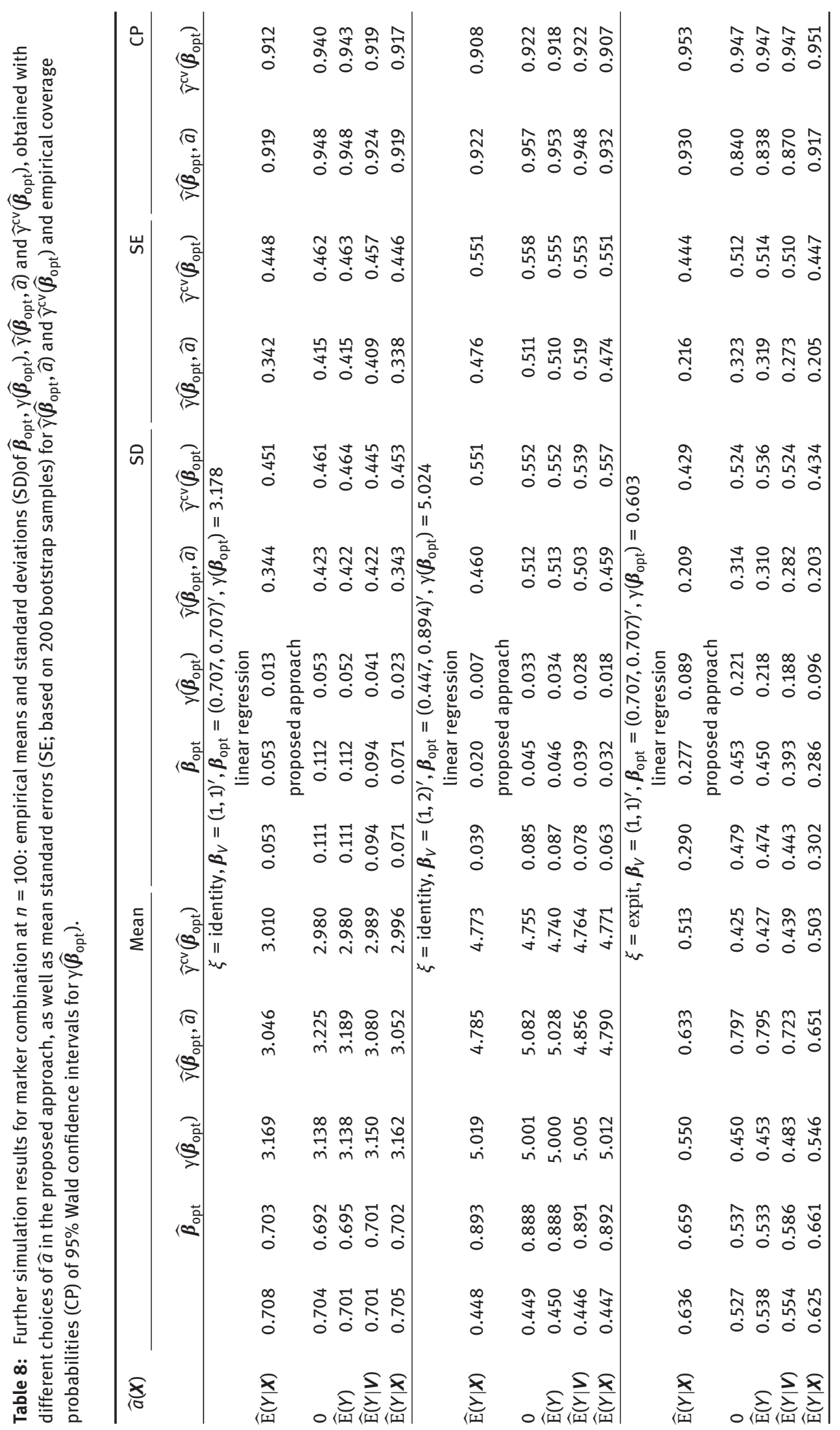




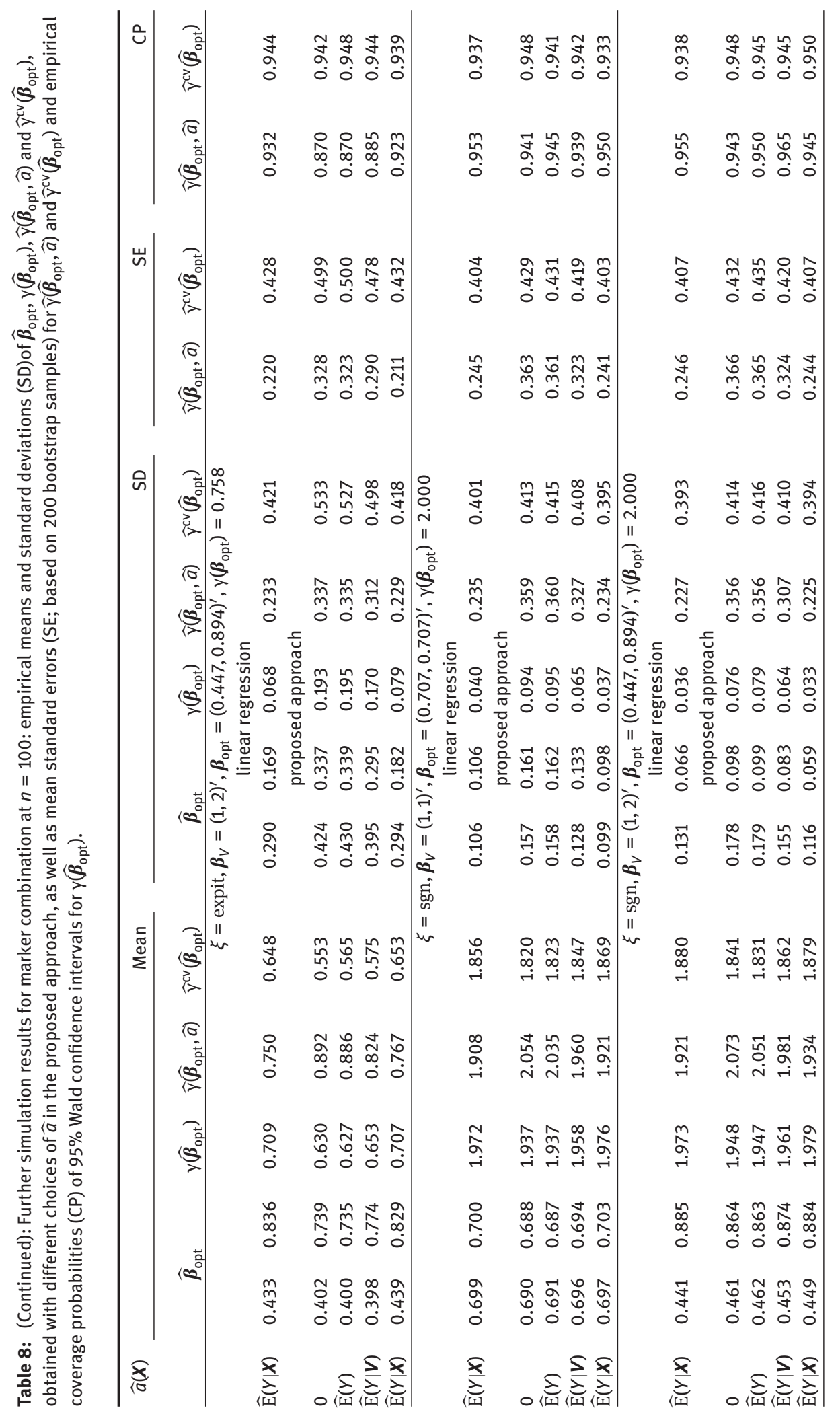

